\title{
LOS NOMBRES DE PILA EN MÉXICO DESDE 1540 HASTA 1950
}

\section{ObservaCiones PRELIMinares}

Igual que las demás modas y costumbres, varía de una generación a otra, y a veces de región en región, la popularidad de los nombres con que bautizamos a nuestros hijos. Tal variación se hace aún más evidente cuando comparamos la onomástica de un solo lugar a través de uno o más siglos.

Recogiendo los copiosos datos biográficos para nuestro fndice geobiográfico de 40,000 pobladores españoles de América en el siglo $x v i^{\mathrm{1}}$, pudimos notar que en el siglo xvi se desconocía la mayoría de los nombres usados en la actualidad (por ej., Ernesto, Gustavo, Joaquin, Roberto, Ramón, Eduardo, Mario, César, Julio, Anibal, Pilar, Dolores, Concepción, Guadalupe, David, Federico) y que en cambio existían otros que hoy han desaparecido casi por completo, como Mayor, Guiomar, García u Ochoa. También observamos la predilección que por determinados nombres de pila mostraba en aquel tiempo una que otra región de España. (Por ejemplo, a principios del siglo xvi acusaban un origen netamente vasco los nombres de pila Ochoa, Ortuño [o Fortún] e fñigo).

Por último, nos llamó la atención la muy poca variedad en los nombres de pila del siglo xvi en comparación con la enorme cantidad que hoy se conoce. Mientras que los españoles del siglo xvi se contentaban casi siempre con un solo nombre de pila, tomado por lo general del Nuevo Testamento (por ej., Juan, Pedro, Francisco, Antonio, Baltasar, Gaspar, Melchior), en siglos posteriores ha surgido la moda de bautizar no sólo con nombres hebreos como Isaac, David, Judith, Sara, Rubén, paganos como Dario, Temistocles, Mario, César, Anibal, Alejandro, Héctor, Aristides, o germánicos como

1 El tomo 1 (La época antillana: 1493-1519) fue editado en 1964 por el Instituto Caro y Cuervo, de Bogotá; el tomo 2 (1520-1539) fue publicado en 1968 por la Editorial Jus, de México; el tomo 3 (1540-1559) está listo para la imprenta desde hace mucho tiempo. La serie completa constará de cinco tomos y registrará la procedencia y otros datos biográficos de más de 60,000 emigrantes del siglo xvr. Cada tomo lleva estudios estadísticos, mapas y gráficos, y diversos indices para facilitar su consulta. 
Ricardo, Alfredo, Raúl, Enrique, Ramona, Carlota, Georgina, sino también con nombres dobles o múltiples. De estos últimos, algunos manifiestan el extraordinario fervor religioso de su época, como por ejemplo Maria de los Angeles Josefa de Jesús Eusebia Juana Bautista de Dios Nepomucena Coleta Sebastiana de Aparicio Hipólita Francisca de Asis de Paula Jerónima y Javiera Luisa Gonzaga Obispa Rita de Casia Ignacia Catalina Teresa Bonifacia, o bien $M a-$ ria Loreto Florencio Modesto Ignacio de Loyola Manuel de Jesús Agustin de la Santisima Trinidad Joaquin Mariano Juan de Dios Antonio de Padua Francisco de Paula Sebastián de Aparicio Nicolás Pedro Nolasco Francisco de Asis Ramón Nonato Pedro Cayetano Lorenzo Manuel Saúl Ismael Gabriel Felipe de Jesuis Luis Gonzaga Rafael Guadalupe Francisco Xabier Francisco de Sales Juan Nepomuceno, nombres que recibieron dos niños mexicanos en 1820 .

\section{Plan de este estudio}

Despertada nuestra curiosidad, no sólo por determinar la cronología de tales cambios de gusto, sino también por ensayar una explicación histórico-cultural, nos propusimos hacer un estudio estadístico basado en los registros de bautismo de una metrópoli hispanoamericana para la que hubiera documentos utilizables, correspondientes a intervalos de unos 20 años desde el siglo Xvi hasta la actualidad. Ahora bien, habiéndose microfilmado ya, en su totalidad, los registros de bautismo del Sagrario Metropolitano de

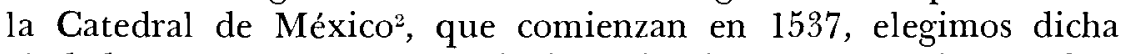
ciudad para nuestro campo de investigación y conseguimos rollos que nos permitieron comparar los nombres de pila usados en 1540 , $1560,1580,1600,1620,1640,1660,1675,1700,1720,1740,1760$, $1780,1800,1820,1830^{3}, 1852,1870,1890,1910,1932$ y 1952 . Durante toda la Colonia, hasta la independencia, se llevaron en México dos series de registros, una para españoles, o sean los criollos, y otra para las castas, o sean los mestizos, mulatos, indios y negros; pero con la independencia se suprimió esta distinción. Para basar nuestro estudio en un cuerpo de datos más homogéneo, decidimos utilizar

2 Trabajo realizado (a costa de los mormones de Salt Lake City, Utah) por la Academia Mexicana de Genealogía y Heráldica en 1954. Los rollos se pueden consultar, previo permiso, en el depósito que custodia la Academia en la calle Tetrazzini, México, D. F. El número total de rollos microfilmados por la Academia, sacados de diversos archivos en todo el pais, ya pasa de $\operatorname{los} 50,000$.

3 Entre 1820 y 1830 median sólo diez años porque queríamos averiguar si con la independencia se produjo un cambio perceptible en el gusto por los nombres de pila. Pero, como después veremos, la independencia no efectuó cambio ninguno en este aspecto. 


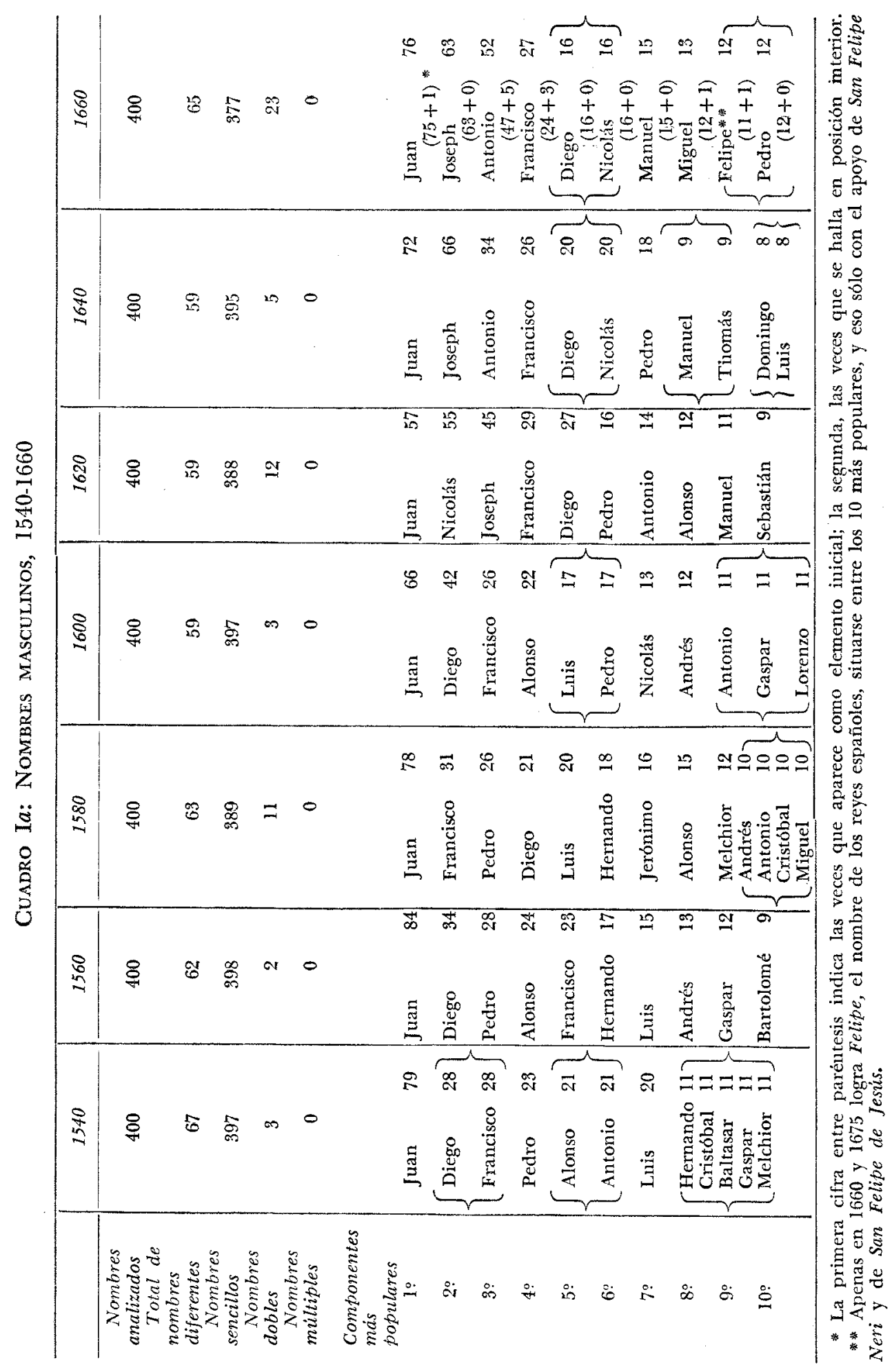




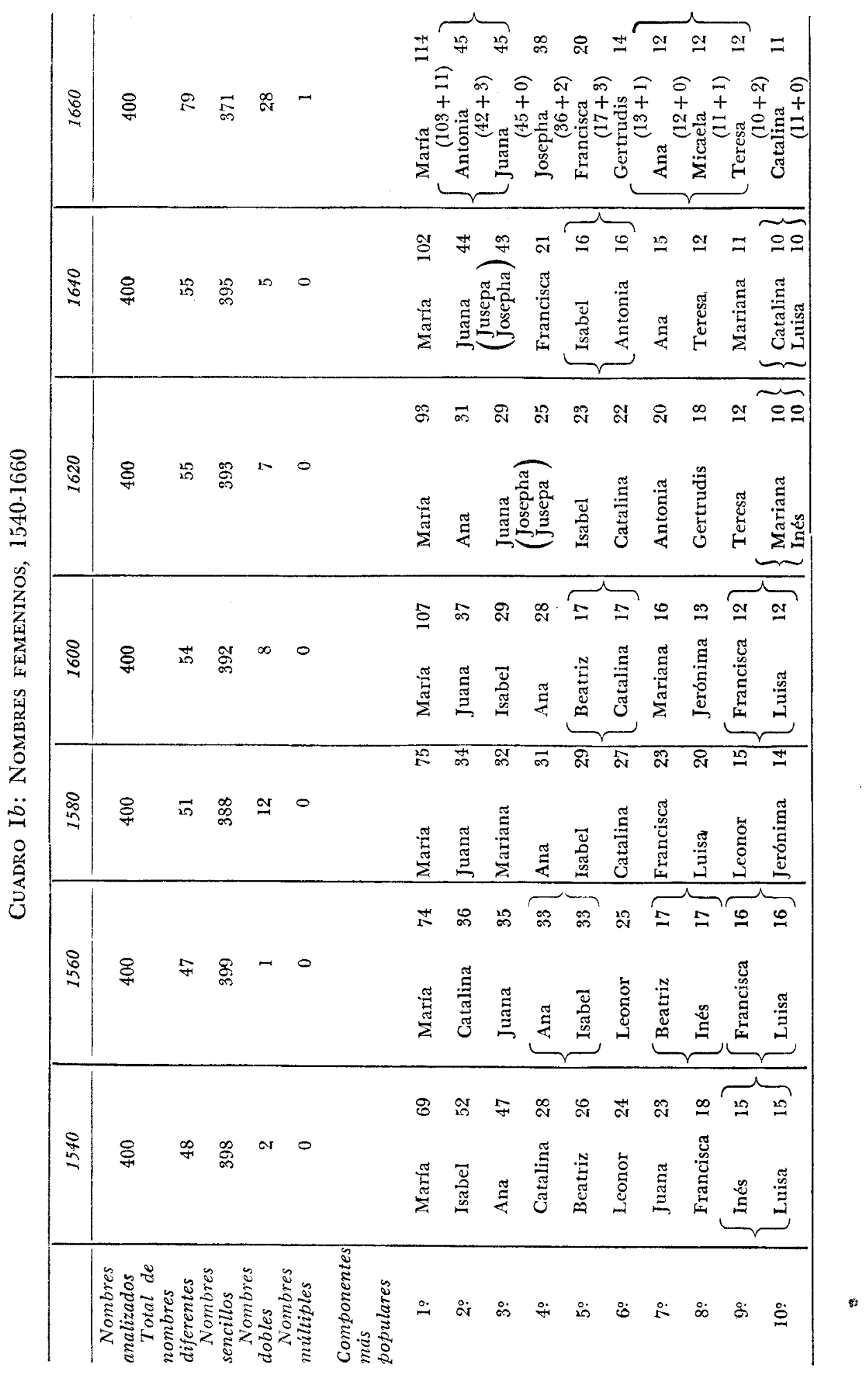


los registros de bautismo de los criollos ${ }^{4}$. Para cada año estudiado analizamos los 400 primeros bautismos masculinos y los 400 primeros femeninos que constaban en los registros, descontando algunos incompletos o bien indescifrables por encontrarse rotos, manchados, tachados o de algún modo mutilados 5 . Elegimos el número de 400 bautismos de cada sexo para dejar aparecer en cada época algunos nombres de menor frecuencia. En cuanto a las variantes ortográficas, las hemos modernizado o regularizado sólo cuando no afectaban la pronunciación, por ejemplo Helena cuenta como Elena, Matheo como Mateo, Luzia como Lucia, Phelipe como Felipe, Ysavel como Isabel, Hierónymo o Gerónimo como Jerónimo.

\section{El Siglo XVI Y LA PRIMERA MITAD DEL Siglo XVII (1540-1660)}

Durante todo el primer siglo estudiado, desde 1540 hasta la segunda mitad del siglo xvn, la onomástica criolla varía muy poco. Nuestros recuentos revelan un gusto sobrio, tradicionalista. Los españoles y criollos se contentan con bautizar a sus hijos con un solo nombre.

No encontramos nombres exóticos ni inventados, y muy pocos compuestos del tipo Juan Bautista o Maria Luisa. Los nombres más comunes hasta 1660 son, para los varones, Juan, Diego, Francisco, Pedro, Antonio, Alonso, Luis, y para las mujeres, Maria, Juana, Isabel, Ana, Catalina y Beatriz. En cada año estudiado los diez nombres más populares representan por sí solos entre el $60 \%$ y el $75 \%$ de la cifra total. El cuadro I muestra los rasgos sobresalientes de la onomástica mexicana hasta mediados del siglo xvn. He aquí algunos comentarios sobre ese cuadro.

Nombres masculinos

El nombre favorito para varones es siempre Juan, aunque hacia fines de la época $(1620,1640)$ comienza a rivalizar con él Joseph, nombre hasta entonces casi inusitado, que en el siglo xvm ha de usurpar el primer rango. Diego, Francisco y Pedro mantienen su popula-

4 En realidad no había ninguna diferencia significativa entre la onomástica criolla y la de las castas, como pudimos comprobar comparando para el año de 1640 los nombres de niños de ambas categorías bautizados en el mismo Sagrario. No encontrando diferencias que merecieran un estudio aparte, desistimos de más comparaciones y nos limitamos a los bautizos de criollos.

${ }^{5}$ Los recuentos que hicimos de nombres de criollos en la ciudad de México, junto con el que hicimos de los nombres de las castas en 1640 y de los criollos de Mérida en 1570-1606 (véase infra), representan un análisis de 19,200 bautizos celebrados en México a través de cuatro siglos de su historia. Esta elevada cifra parece más que suficiente para garantizar la validez de las conclusiones que sacamos. 
ridad a través de toda la época, mientras que el terreno perdido por Luis y Hernando -después de 1640 casi desaparecen- lo van ganando Antonio, Joseph y (sobre todo en 1620) Nicolás. Los Reyes Magos, que al principio gozaban de cierta boga (Gaspar 11, Melchior 11 y Baltasar 11 en 1540), para 1660 habían desaparecido totalmente. Alcanza repentino favor en 1580 el nombre de Jerónimo.

Aunque un muestreo de sólo 400 nombres masculinos no pretende registrar todos los usados en determinado año, sobre todo los muy esporádicos, sí puede interesar al lector notar el año en que un nombre ha adquirido una frecuencia estadística suficiente para figurar en nuestro recuento.

Así encontramos por primera vez, en el recuento de 1560: Asencio 1, Cebrián 1, Fabián 1, Llorente 1, Ochoa 1, Salvador 4, Simón 1, Vasco 1 y Vicente 1. En el de 1580: Alexandre 1, (Juan) Bautista 1, Crispiniano 1, Gómez 1, Iñigo 1, Juste 1, Marco 1, Pascual 1, Roque 1 y Santos 1. En el de 1600: Adriano 2, Bernardo 1, Ignacio 1, Jacinto 7 y Vicencio 1. En el de 1620: Alfonso 3, Bartolo 1, Clemente 2, (Luis) Desiderio 1, Hermenegildo 2, Ildefonso 1, Isidro 1, Marcelino 1, Reymundo 1, Serafin 1 y (Francisco) Xavier 1. En el de 1640: Alberto 1, Alexandro 1, (Leonís) Angel 1, Angelo 1, Joachin 1, Laureano 1, Leonis (Angel) 1, Mario 2, Raphael 1 y Ventura 1. En el de 1660: Eligio 1, Félix 1, Fulgencio 1, Gervasio 1, Michael 1, Onofre 1 y Ramón 1. Por otra parte, entre los nombres masculinos del siglo xvi que tienden a caer en desuso en el transcurso del xvn apuntamos Alvaro, Bernaldino, Cosme, Fabiän, Garcia, Gaspar, Gonzalo, Hernando, Juliản, Lázaro, Leonardo, Lope, Nuflo, Ochoa, Pablo, Ruy, Sancho, Serván, Silvestre y Vasco.

\section{Nombres femeninos}

Aunque en aquellos tiempos ningún nombre de mujer rivalizaba en popularidad con el de María, la madre de Jesús, se buscará en vano, entre los nombres de niñas nacidas en México hasta fines del siglo xvn, esas alusiones a las advocaciones de la Virgen tan características de la onomástica de los siglos xviri y xix. Nada de Concepción, de Encarnación, de Carmen, de Consuelo, de Dolores, de Mercedes, de Rosario o de Soledad. El repertorio de nombres femeninos resulta todavía más limitado que el de los masculinos. Además de Maria, nombre que recibieron en dos recuentos más del $25 \%$ de todas las niñas bautizadas, siguen siempre populares durante esta época Isabel, Ana, Juana, Catalina, Francisca y Luisa. Los nombres de Beatriz, Leonor e Inés, posteriormente tan queridos de los poetas románticos por su sabor medieval, comienzan a declinar después de 1600. En cambio surgen Jerónima, Mariana, Antonia, Teresa (sin duda con motivo de la canonización, en 1622, de Santa Teresa de Ávila), y a partir de 1620 Josepha (o Jusepa) y Ge(r)trudis. La creciente popularidad de Josepha coincide con la del masculino Joseph.

Es bastante común la derivación a partir de nombres masculinos. Así encontramos Baltasara, Bernabela, Blasia, Blasina, Crispiniana, 
Damiana, Dominga, Felipa, Íniga, Jacinta, Jacoba, Joachina, Josepha, Lorenza, Lorenzana, Manuela, Mathiana, Melchiora, Nicolasa, Pascuala, Sebastiana, Simona, Thomasa.

Aparecen por primera vez, en el recuento de 1560: Bárbola 1, Elena 3, Escolástica 1, Florencia 1, Gregoria 1, Hipólita 1, Jimena 1, Josepha 1, Mayor 1, Paula 1, Sabina 1, Teresa 1, Tomasina 1. En el de 1580: Agueda 2, Blasina 1, Brigida 1, Cristina 1, Iñiga 1, Lorenza 1, Lorenzana 1, Marcela 1, Susana 1, Vitoria 1. En el de 1600: Clemencia 1, Estebania 1, Estefania 1, Micaela 1, Petrona 1, Rosarda 1. En el de 1620: Angelina 2, Apolonia 1, Bárbara 1, Bernarda 1, Ge(r)trudis 18 (!), Hernandina 1, Leonora 1, Tomasa 1, Viviana 1. En el de 1640: Bernabela 2, Faustina 1, Feliciana 1, Jacinta 2, Leonis 1, Manuela 2, Ma thiana 1. En el de 1660: Baltasara 1, Blasia 1, Catherina 1, Cecilia 1, Dominga 1, Elugarda 1, Joachina 1, Theodora 1. Entre los nombres femeninos del siglo xvr que no pasan al xvn se cuentan $A g u e d a, A l d o n$ za, Beatriz, Bernaldina (o Bernardina), Casilda, Elvira, Guiomar, Inés, Jimena, Leonor, Marina, Mayor, Mencia y Violante.

Con el deseo de averiguar si podía haber notables diferencias de gusto, por los mismos años, entre una y otra ciudad de la Nueva España, hicimos un análisis de los nombres de criollos bautizados en Mérida (Yucatán) en el último cuarto del siglo xvi. Para reunir 800 nombres en una ciudad tan pequeña como lo era por entonces la capital yucateca, nos vimos obligados a aprovechar todos los bautizos de criollos registrados en el Sagrario de Mérida entre 1570 y 1606. Estadísticamente encontramos una correspondencia bastante precisa entre los nombres masculinos recopilados en Mérida entre 1570 y 1606 y los que se dieron en la ciudad de México en 1580:

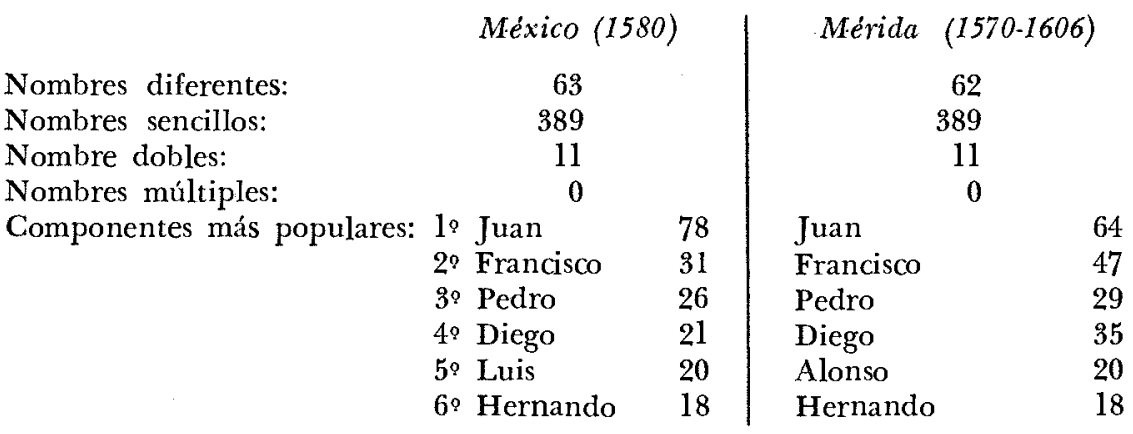

En el quinto lugar presenta Mérida Alonso en vez de Luis, pero en lo demás muestra las mismas preferencias generales. Hay muchas diferencias, pero todas son ligeras. Nombres esporádicos que encontramos en Mérida pero todavía no en la capital son Alejo 1, Bonifacio 1, Guillermo 1, Gutierre 1, Onofrio 1 y Velasco 1.

La correspondencia entre los nombres femeninos empleados en México y en Mérida es menos exacta: 


\begin{tabular}{|c|c|c|c|c|}
\hline & \multicolumn{2}{|c|}{ México (1580) } & \multicolumn{2}{|c|}{ Mérida (1570-1606) } \\
\hline Nombres diferentes: & 51 & & & \\
\hline Nombres sencillos: & 388 & & & \\
\hline Nombres dobles: & 12 & & & \\
\hline Nombres múltiples: & 0 & & & \\
\hline Componentes más populares: & 1: María & 75 & María & 75 \\
\hline & $2^{\circ}$ Juana & 34 & Ana & 47 \\
\hline & 3: Mariana & 32 & Juana & 38 \\
\hline & $4^{\circ}$ Ana & 31 & Isabel & 36 \\
\hline & $5^{\circ}$ Isabel & 29 & Catalina & 34 \\
\hline & $6^{\circ}$ Catalina & 27 & Francisca & 27 \\
\hline & 7\% Francisca & 23 & Beatriz & 21 \\
\hline
\end{tabular}

En la capital, entre 1560 y 1580 , había perdido repentinamente en popularidad el nombre de Beatriz y nacido, también de repente, el de Mariana. Como vemos, ninguna de las dos innovaciones se documenta todavía en Mérida, lejana ciudad provinciana que tardará algún tiempo en reaccionar a los cambios iniciados en la capital. Por otra parte encontramos por primera vez en Mérida Agada 1, Dorotea 2, Eufemia 1, Fulsina 1, Justina 1 y Mathea 1.

Del Último cuarto Del siglo XViI AL Siglo XIX (1675-1890)

El culto mariano.-En mayo de 1965 apareció en Cuadernos del Idioma (Buenos Aires, núm. 1, 9-16) un corto pero muy interesante artículo de Ramón Menéndez Pidal titulado "Onomástica inspirada en el culto mariánico". En él observó don Ramón que en España la onomástica marial debe su auge al movimiento teológico, iniciado en 1615 por los carmelitas de Sevilla, en pro del dogma de la Inmaculada Concepción. Inspirados en una abundante literatura doctrinal en torno a este asunto, comienzan a aparecer esporádicamente en los registros de bautismos de ciudades como Madrid y Sevilla, adjuntas al nombre de Maria, algunas advocaciones como Maria de la Concepción y Maria de la O. Pero tanto las advocaciones referentes a misterios mariales como el uso de nombres alusivos a santuarios o imágenes veneradas (Carmen, Pilar, Loreto, Guadalupe) empiezan a tomar incremento sólo a partir de 1665, año en que el papa Alejandro VII promulga oficialmente la doctrina de la Inmaculada Concepción. En Madrid encuentra don Ramón los primeros ejemplos de Maria del Carmen en 1667, del Rosario 1673, de los Dolores 1676, del Pilar 1677, de Loreto 1684, de Guadalupe 1689, de las Mercedes 1690. En cambio Maria de la Soledad lo encuentra primero en Sevilla (1703).

Según parece por los datos que hemos analizado nosotros para México, la Nueva España siguió con cierto retraso las tendencias registradas en la Península. Las advocaciones comienzan repenti- 
namente en 1675 (el primer año analizado después de promulgarse la doctrina de la Inmaculada Concepción) y alcanzan su mayor profusión entre 1800 y 1852 , año este último en que contamos 260 advocaciones a la Virgen entre los 400 nombres femeninos estudiados. A partir de 1870 el número de advocaciones baja perceptiblemente, registrándose en nuestro análisis de 1952 no más de 79 en los nombres femeninos, y en los masculinos sólo 3.

Ya para 1675 se dan en México Asunción, Concepción, Encarnación, Guadalupe, Natividad, Rosario y Maria de la O. En 1700 encontramos por primera vez Asunción, Candelaria, Dolores ( 26 veces!), Loreto y Nieves, en 1720 Carmen y Carmelo, en 1740 Luz ( 7 veces en los femeninos, 2 veces en los de varón), en $1760 \mathrm{An}$ geles y Rosario, en 1780 Pilar y Soledad, en 1820 Refugio. La advocación predilecta en México fue y sigue siendo todavía la de Guadalupe, venerada en México desde su aparición allí en $1531^{6}$.

La devoción a la Virgen de Guadalupe, patrona de México, alcanzó su apogeo en vísperas de la Guerra de Independencia: en el recuento de 1800 aparece este nombre nada menos que 68 veces. Todavía en 1852 contamos 59 alusiones a dicha Virgen, y en años recientes, entre los nombres de mujer, es la única advocación que todavía se repite con frecuencia. Aunque apuntamos unas 30 advocaciones diferentes que se han usado alguna vez en la ciudad de México, solamente seis han gozado de favor popular por mucho tiempo: Guadalupe, Dolores, Soledad, Concepción, Luz y Carmen. Entre los nombres de varón Guadalupe fue la única advocación que mantuvo por largo tiempo su popularidad, pero aun ésta cayó en desuso después de la Revolución mexicana.

María de la $O$ aparece 6 veces como nombre de mujer en 1675, 2 veces más en 1700, después ya no ${ }^{7}$ Maria de los Ángeles alcanzó

6 Para satisfacer nuestra curiosidad hicimos aparte un análisis exhaustivo de todas las advocaciones de la Virgen registradas en los libros de bautizos de españoles en el Sagrario Metropolitano de México entre 1665 y mayo de 1672, o sea un período de más de siete años. Las pocas advocaciones que hallamos se dan exclusivamente en nombres de mujer: 5 Maria de la $O$ (27/XII/1665, 29/XII/68, 30/XII/68, 6/1/70, 29/XII/71), 2 Maria de la Candelaria (7/II/66, 15/11/71), 2 Maria de los Ángeles (8/1II/66, 2/III/71), 2 Maria de la Concepción (I5/VI/66, 21/XII/70), 3 Maria de Guadalupe (10/IX/67, 23/IX/71, 8/1/72), I Maria de la Purificación (13/11/68), 5 Maria de la Encarnación $(7 / 1 \mathrm{~V} / 69,3 / \mathrm{III} / 70,6 / \mathrm{IV} / 70,7 / \mathrm{IV} / 70,6 / \mathrm{IV} / 7 \mathrm{l})$ y 1 Maria de la Asunción (26/V/72). Además, hallamos 2 Maria de Jesús $(2 / \mathrm{V} / 68,23 / 1 / 71), 2$ Maria de la Cruz (14/V/68, 18/V/70) y 1 Maria de la Trinidad (3/VI/68). Total: 21 advocaciones (26 si contamos Jesús, Trinidad y Cruz), o sea un promedio de 3 por año.

${ }^{7}$ La antigüedad de dicha advocación la confirman dos muchachas sevillanas, Maria de la $O$ y Ana de la $O$, que emigran a América con sus madres, la primera a México en 1555, la otra al Perú en 1559. Véase el Catálogo de pasajeros a Indias, t. 3, No. 3254 y No. 4430. 
una corta boga entre 1820 y 1852 , Consuelo y Esperanza entre 1910 y 1930, Lourdes en 1952, Mercedes entre 1800 y 1852. Encarnación, Loreto, Pilar y Rosario aparecen después de la primera vez en la mayoría de los años analizados, pero con baja frecuencia. Aunque no faltaron en años pasados en los nombres de varón (sobre todo entre 1800 y 1852), las advocaciones a la Virgen son esencialmente un rasgo de la onomástica femenina.

Estrechamente relacionadas con las citadas advocaciones de la Virgen, rasgo sobresaliente de la onomástica mexicana entre 1675 y 1852, se destacan otros tres fenómenos: el culto de los santos, el culto de la Sagrada Familia, el cual alcanzó proporciones poco menos que increíbles hacia la época de la independencia, y el gusto por los nombres múltiples, de entre 4 y 10 o más elementos.

Examinemos primero el extraordinario culto de la Sagrada Familia, el cual comienza a registrarse por 1700 cuando empiezan su curiosa trayectoria tanto el nombre de María como el de su esposo Joseph. Va creciendo la popularidad de estos dos nombres hasta tal extremo, que de todas las niñas bautizadas en 1800, nada menos que $92 \%$ recibieron el nombre de Maria, y $34 \%$ el de Josephas.

Entre los varones, en aquel mismo año de $1800,78 \%$ se 11 maron Joseph y $40 \%$ recibieron como nombre secundario Maria. $\mathrm{Y}$ esto sin contar los derivados Mariano y Mariana, que alcanzan su máximo en 1740 pero que siguen usándose (sobre todo $\mathrm{Ma}$ riano) hasta mediados del siglo xIx, ni el nombre de Jesús, muy popular en los bautizos de ambos sexos entre 1800 y 1852 (sobre todo en 1830), ni los nombres Manuel y Manuela, que alcanzaron su apogeo en 1740 y 1800 respectivamente. Según se verá por el cuadro II, el culto a la Sagrada Familia llegó a su colmo en 1800, se mantuvo casi al mismo nivel durante la Guerra de Independencia, y luego comenzó a declinar.

s Gutierre Tibón, Diccionario etimológico comparado de nombres propios de persona, México, 1956, p. 352, basándose en el Oxford dictionary of English christian names, afirma que "durante muchos siglos el nombre de la Virgen María se consideró demasiado sagrado para usarlo como nombre de pila. En España se emplearon en sustitución nombres de sus advocaciones o atributos, como Pilar, Socorro, Concepción, Refugio, Amparo, Dolores, Soledad, etc." Esta última afirmación no concuerda con la realidad, la cual nos revela que el nombre de Maria ya había alcanzado una extraordinaria popularidad más de un siglo antes de aparecer advocaciones como Dolores o Pilar. Y aun más, cuando éstas empezaron a usarse no sustituian a Maria, sino que se le agregaban (por ej. Maria de los Remedios, María de la Soledad). Sólo en tiempos recientes comienzan a figurar Soledad, Dolores o Concepción como nombres independientes, sin duda para distinguir entre tanta Maria. Así también, de Maria de Jesuis debe haberse formado (Maria) Jesusa y por fin el hipocorístico Chucha. (Véase nuestra última nota). 
Cuadro II: El culto a la Sagrada Familia en la onomástica mexicana

\begin{tabular}{|c|c|c|c|c|c|c|c|c|c|c|c|c|}
\hline & \multicolumn{6}{|c|}{ FEMENINOS } & \multicolumn{6}{|c|}{ MASCULINOS } \\
\hline & Maria & Mariana & Josepha & Manuela & Jesús, de ${ }^{*}$ & $\begin{array}{l}\text { Nombres } \\
\text { múltiples }\end{array}$ & Josep $(h)$ & Mariano & Maria & Manuel & Jesuis, de* & $\begin{array}{l}\text { Nombres } \\
\text { múltiples }\end{array}$ \\
\hline 1660 & 114 & - & 38 & 8 & - & 1 & 63 & - & - & 15 & - & 0 \\
\hline 1675 & 123 & 2 & 16 & 14 & 2 & 8 & 52 & 一 & - & 24 & - & 6 \\
\hline 1700 & 172 & 7 & 61 & 34 & 2 & 68 & 122 & 一 & - & 43 & - & 47 \\
\hline 1720 & 162 & 7 & 84 & 35 & 1 & 89 & 133 & 10 & - & 48 & 1 & 79 \\
\hline 1740 & 258 & 15 & 164 & 43 & 5 & 237 & 245 & 93 & 17 & 43 & 1 & 192 \\
\hline 1760 & 282 & 13 & 186 & 28 & 4 & 295 & 279 & 62 & 53 & 56 & 3 & 238 \\
\hline 1780 & 329 & 13 & 149 & 29 & 4 & 335 & 269 & 39 & 68 & 34 & 1 & 297 \\
\hline 1800 & 367 & 9 & 137 & 22 & 37 & 377 & 313 & 41 & 161 & 59 & 20 & 367 \\
\hline 1820 & 360 & 5 & 113 & 30 & 48 & 358 & 309 & 31 & 113 & 29 & 39 & 352 \\
\hline 1830 & 353 & 7 & 79 & 13 & 62 & 358 & 276 & 21 & 48 & 31 & 67 & 332 \\
\hline 1850 & 173 & 5 & 36 & 13 & 49 & 315 & 190 & 8 & 54 & 32 & 53 & 287 \\
\hline
\end{tabular}

* E1 nombre de Jesús no aparece como elemento inicial hasta el recuento de 1852. 
El culto de los santos ${ }^{9}$.-Mientras que las advocaciones marianas son mucho más frecuentes en los nombres femeninos que en los masculinos, con los nombres de los santos suele ocurrir lo contrario. Por ejemplo, entre los años de 1700 y 1720 Francisco Xavier aparece 11 veces en los femeninos (generalmente feminizado en Francisca Xaviera), pero en los masculinos 36 veces. Entre 1800 y 1850 figura Luis Gonzaga 74 veces en los femeninos, pero en los masculinos 139. En México fueron sólo cuatro los santos cuyos nombres se repitieron con frecuencia entre 1675 y 1852: Luis Gonzaga (canonizado en 1726), Juan Nepomuceno (canon. en 1729), Francisco de Paula (canon. en 1519) y (ya desde 1660) Francisco Xavier (canon. en 1623). Todos los demás (por ejemplo Francisco de Asis, Pedro de Alcántara, Juan de Dios, Vicente Ferrer, Felipe Neri, Pedro Nolasco, Francisco de Sales, Santa Teresa, Sebastián de A paricio) salen sólo esporádicamente, aunque sospechamos que muchos Ignacios y Vicentes (con sus formas femeninas correspondientes) pueden aludir a San Ignacio de Loyola y San Vicente Ferrer.

Muchas veces la canonización de un santo no es la causa sino el resultado de un creciente entusiasmo popular. En el caso de Santa Gertrudis, monja y escritora alemana del siglo xiII, una larga e insistente devoción popular logró que el papa Inocencio XI la incluyera al fin en el martirologio romano en 1677, y que luego el papa Clemente XII la mandara venerar en todo el mundo católico.

Nuestras cifras para el nombre de Gertrudis en México muestran muy claramente la cronología:

\section{Gertrudis}

$$
\begin{aligned}
& 1620-18 \text { veces } \\
& 1640-5 \text { veces } \\
& 1660-14 \text { veces } \\
& 1675-54 \text { veces }(!)
\end{aligned}
$$

(El Papa accede en 1677 al clamor popular)

$$
\begin{aligned}
& 1700-43 \text { veces } \\
& 1720-23 \text { veces } \\
& 1760-32 \text { veces } \\
& 1780-57 \text { veces }
\end{aligned}
$$

Y luego, cediendo su popularidad a otros santos: 1800-10 veces.

El gusto por los nombres múltiples, o sea de tres o cuatro elementos hasta diez, doce, y aun más, está directamente vinculado con el deseo de invocar la protección divina o sobrenatural enco-

9 Santos: Solamente los identificamos como tales si aparecen con el título de santo, con nombre y apellido (por ej. Luis Gonzaga), o con el apellido solo (Gonzaga). Por la pluralidad de santos que llevaron el mismo nombre sería demasiado inseguro basar una identificación en el solo nombre de pila. 
mendando al recién nacido a uno o más de los santos o a algún miembro de la Sagrada Familia. Abundan a partir de 1700 nombres como Joseph Maria de Jesús, Maria Guadalupe Josefa de Jesús, José Maria Ponciano Bruno Juan Nepomuceno Luis Gonzaga, José Mariano Joachin Luis Gonzaga Francisco de Paula, Luis Gonzaga José Maria Abraham Ciriaco Nepomuceno Francisco de Paula, y hasta Maria de la Concepción Manuela Josepha Joachina Ana Rafaela Ramona Cleofas Demetria Francisca de Paula Luisa Gonzaga de la Santisima Trinidad. Entre 1800 y 1820 los nombres de pila de ambos sexos tienen un promedio de cuatro componentes cada uno, sin contar artículos ni preposiciones. Después de 1852 comienzan a declinar, junto con el culto de la Sagrada Familia y de los santos, la proporción y el tamaño de los nombres múltiples. El cambio se realiza más rápidamente en los nombres de varón que en los femeninos, los cuales en 1890 denuncian todavía una breve pero intensa resurgencia de las advocaciones marianas y por consiguiente una proporción más elevada de nombres múltiples.

A continuación ofrecemos algunos comentarios sobre el cuadro III, que presenta los recuentos hechos para el período 16751890 .

Nombres masculinos

Entre 1660 y 1675 empiezan a aparecer nombres que atestiguan el culto de los santos, pero hasta 1720 no son más que esporádicos.

1720: Hay pocas advocaciones de la Virgen. Se nombra 3 veces la Encarnación, 2 veces Guadalupe, y sendas veces Asunción, Candelaria, Carmelo, Dolores y Loreto. Entre los santos se nombran: San Ignacio 32, (San Francisco) Xavier 18, Juan de Dios 7, Santiago 5, Santa Ana 3, de la Rosa 2, Pedro Nolasco 2, (Luis) Gonzaga 1. Nombres poco usuales que figuran aquí: Agraciano 1, Astacio 1, Atenógenes 1, Buenaventura 1, Claudio 1, Estanislao 1, Fulgencio 1, Higinio 1, Hilario 1, Perfecto 1, Rudisendo 1, Sabino 1, Theobaldo 1.

1740: Ahora, con el gran auge del culto de la Sagrada Familia, comienza a aparecer por primera vez en los nombres de varón, al lado del derivado Mariano, el mismo nombre de Maria. Joseph se nombra 245 veces (130 como elemento primero), Mariano 93 y Maria 17. Hay 43 Manuel, pero sólo 1 Jesús. Se nombra 3 veces la Santísima Cruz. También es popular el nombre de la (Santísima) Trinidad 14. Son todavía pocas las advocaciones marianas: Encarnación 4 y Loreto 4, Guadalupe 3, Luz 2, Dolores 1.-Se cita un buen número de santos: Ignacio (¿de Loyola?) 22, (Juan) Nepomuceno 4, (Juan) Bautista 2, (Pedro) Nolasco 2, Francisco de Paula 2, Santa Rosa 2, y sendas veces Santa Inés, San Miguel, San Bartolomé, Santiago, Santa Rita, Santa Rosalia, Santa Marta y Vidal.-Nombres poco usuales: Cresiano 1, Leonicio 1, Hilarión 1, Claudiano 1, Sixto 1, Narciso 1, Quirino 1, Venicio 1, Felis 1, Veronio 1, Liborio 1, Cástulo 1, Inesiforo 1, Florencio 1. 
1760: Advocaciones y santos: Además de Jose( $p h)$ 279, Mariano 62 y Maria 53, salieron Ignacio 73, (M. de la) Luz 7, (Juan) Nepomuceno 6 y (la Santísima) Trinidad 6, Loreto 4, Dolores 3, Guadalupe 3, Jesús 3, (Pedro) Nolasco 3, Justo Rufino 3 (más Sta. Justa Rufina 1, Rufino 1, y Justo Joseph Rufino 1), Dios 2, Natal 2, más sendas veces Asunción, Cruz, Francisco de Paula, Pioquinto, Regio, Roncabo, Rosario, de los Reyes; Sta. Getrudis, Sta. Rosa, Vidal, Vito, Xavier y la Purisima Sangre de Cristo.

1780: Sigue el culto de la Sagrada Familia y de los misterios marianos: José 269 (222 veces como elemento primero), Maria 68, Mariano 39, Manuel 34, Maria de Guadalupe 6, Maria de la Santa Cruz 3, de los Angeles 2, de Jesús 1, del Monte 1.-Se nombra 4 veces la Santísima Trinidad. Entre los santos, el más nombrado es (San Juan) Nepomuceno 20. También aparecen (San) Vito 6, Francisco de Sales y Juan de Dios 3, Pedro de Alcántara y Santa Teresa 2, Luis Gonzaga 1, Santa Elena 1, Santa Lugarda 1, Santa Rita 1, Santiago 1, y Remigio de los Dolores 1.-Son formas derivadas del femenino Brigido 1, Catarino 1 y (si no es un error por Pedro) Petro 1.-Aparece 3 veces Francial, 2 veces Leónido, y una Nucio (¿por Nuncio?).

1800: El culto de la Sagrada Familia ha tomado un fuerte impulso. Más del $60 \%$ de todos los varones reciben José como nombre primero y otro $16 \%$ como nombre secundario; $40 \%$ de los varones reciben además el nombre de Maria. Sale 59 veces Manuel, 20 veces Jesús, 16 veces (la Sma.) Trinidad, 5 veces la Santa Cruz, 3 veces el Corazón de Jesús, una vez Bethlén.-Además de los 161 Maria, se nombra mucho Maria de Guadalupe 23, la cual como patrona de Hispanoamérica y de México parece alcanzar su mayor popularidad en vísperas de la Guerra de Independencia. Salen también (el Buen) Loreto 10, Carmen 6, Dolores 4, Soledad 3, Luz 2, y sendas veces Angeles, Concepción, Remedios, Rosario y (advocación muy infrecuente) Maria de los Desamparados.-Entre los santos goza de una devoción extraordinaria Luis Gonzaga, el cual se nombra nada menos que 70 veces. Se citan 23 veces Juan Nepomuceno, 15 veces (Francisco de) Paula, 7 veces Juan de Dios, 4 el santo mexicano Sebastián de Aparicio, 4 Juan Evangelista, 3 Antonio Abad, 2 Felipe Neri y los cinco Señores, y sendas veces Sta. Brigida, Vicente Ferrer, Sta. Inés, Luis Obispo, Nicolás Obispo, Pioquinto (= el papa Pío V, canonizado en 1712), Francisco de Sales y Francisco Solano.-Del Viejo Testamento llegan Abrahán 2, Isaac 1, Ismael 3 y Jaeobo 1.-Derivan de nombres femeninos Catalino 1, Ca tarino 1, Cecilio 1, Cristino 1, Dorotheo 1, Felicito 1, Genovevo 2, Magdaleno 1, Teclo 1 y Úrsulo 1.-Un nombre muy largo pero representativo del gusto de la época es Manuel María Guadalupe José Joachin Antonio Abad Juan Nepomuceno Felipe Neri Luis Gonzaga Sebastián de Aparicio Juan.

1820: De 400 niños bautizados, 273 recibieron José como nombre primero, y otros 36 como nombre secundario, o sea más del $77 \%$.-La devoción a varios santos se documenta en repetidas alusiones a Luis Gonzaga (así 37 veces, más 11 Luis solos), a Francisco de Paula (28 
veces, más otros 23 Francisco de los cuales algunos pueden referirse al de Paula), y a (Juan) Nepomuceno (20 veces). Ignacio de Loyola aparece 2 veces, pero Ignacio por sí solo 46 veces más). Lo mismo pasa con Vicente Ferrer (Ferrer 2 veces, Vicente 23 veces). (Francisco) Javier sale 7 veces, (José) Bruno 4, Francisco de Sales 2 veces, lo mismo que (Pedro) Nolasco y (Francisco) de Borja. Sendas veces se nombran Francisco de Asis, Francisco de Posadas, Pedro de Alcántara, Antonio de Padua, (Juan) Climaco y Pioquinto.-Se derivan de nombres de mujer Eleno, Rito, Teclo, Margarito, Brígido, Catarino, Rosalio, Rosalino, Epifanio y tal vez Marcio.-i400 niños recibieron un total de 1642 nombres, o sea un promedio de 4 cada uno!

1830: La Sagrada Familia sigue siendo mentada por todos lados: José 276, Jesús 67 (pero nunca inicial), María 48 (tampoco inicial), Trinidad 33, Manuel 31, Mariano 21 (inicial 4 veces), Guadalupe 11 (una vez inicial), Dolores 8, los Angeles 8, Cruz 6, Carmen 5, Concepción 5, Pilar 5, Loreto 3, Merced 3, Refugio 3, Luz 2, Nieves 2, Amparo 1, Asunción 1, Encarnación 1, Felicitas 1, Remedios 1, Rosario 1. Aparece dos veces el nombre del Corazón de Jesús, una vez el Dulce Nombre de Maria. En cuanto a la advocación de santos, ocupa el primer lugar (Luis) Gonzaga 20, el segundo (Francisco de) Paula 18.-Entre los nombres poco usuales, algunos ya paganos, notamos Eduvige 2, Atenógenes 1, Nexy 1, Atilano 4, Fortino 1, Cornelio 1, Sóstenes 2, Albendio 1, Platón 2, Benicio 2, Eutimio 2, Espiridión 1, Fructuoso 1, Salomé 1, Serapio 2, Avelino 4, Leónides 1, Prajedis 2, Narciso 2, Macario 1, Librado 1, Emigdio 1, Melquiades 1, Atioco 1, Zenobio 2, Tribonio 1, Herculano 3, Bruno 3, Alvino 3, Lino 1, Nabor 2, Sabás 3, Zacarias 5, Telésforo 1, Cleofas 1, Conrado 1, Dario 2, Fausto 3, Amado 6, Cristíno 1, Proto 1.-Nombres femeninos masculinizados: Brígido 2, Catarino 2, Eleno 1, Lauro 2, Magdaleno 2, Margarito 1, Regino 2, Rosalio 3, Rosalino 1, Victorio 1, Zenobio 2.-Entre los nombres largos se destacan Ignacio José Joaquin de Jesús Pedro Advincula Francisco de Asis Gonzaga Antonio de Padua Andrés Avelino Magdaleno Hipólito Rafael Juan Nepomuceno, y Joaquin Mariano José de Jesús Pascual Nicolás Florentino Ponciano Vicente Ferrer Brigido Ramón Ignacio.

1852: La Sagrada Familia está representada por 190 José, 54 Maria, 53 Jesús (3 de ellos ya en posición inicial), 32 Manuel, 4 Corazón de Jesús, la Preciosa Sangre (de Cristo) 1 y las Cinco Llagas 1. Se nombra 28 veces (la Sma.) Trinidad, 2 veces como nombre primero de varón. En cuanto a las alusiones a los misterios de la Virgen, son cuatro veces más numerosas entre los nombres femeninos que entre los masculinos. Sólo dos se repiten con alguna frecuencia en este año: Guadalupe 20 y Soledad 13.-De los santos, los más citados son Francisco de Paula 22, Luis Gonzaga 12, Juän Nepomuceno 9 y Juan de la Cruz 9. También se mencionan Francisco de Paula 5, Francisco Xavier 4, Pedro de Alcántara 3, Sta. Teresa 2, Sta. Bárbara y Juan Capistrano 2, Francisco de Asis 1, Felipe Neri 1, Homobono 1, Sta. Rosa 1, Vicente Ferrer 1, Sta. Rita 1, Borja 1, Bruno 1.-De nombres femeninos derivan Adelaido 1, Bárbaro 1, Brígido 1, Casildo 1, Catalino 1, Catarino 2, 
Cecilio 3, Celestino 1, Clariso 1, Corino 2, Doroteo 1, Eulalio 2, Lauro 4, Magdaleno 1, Regino 2 y Tereso 1.-Es notable la variedad de elementos que figuran en estos 400 nombres. Algunos son raros, otros nuevos. Además de los derivados de femeninos ya citados, merecen señalarse Abundio 2, Alvino 1, Arcadio 2, Aristero 1, Aurelio 6, Calixto 3, Carino 1, Cenobio 2, Ciro 1, Colombo 1, Crisanto 1, Delfin 1, Demetrio 1, Eudivio 1, Felo 1, Femato 1, Flavio 1, Fortino 2, Gabino 1, Gamero 1, Genaro 2, Gión 1, Gorgonio 4, Hermelio 1, Legonio 1, Leonildes 1, Lino 3, Lacerencio (sic) 1, Macario 2, Mauro 1, Melesio 1, Melquiades 1, Nativo 1, Pio 2, Pronto 1, Próspero 4, Roselino 1, Sabad 1, Saórino 1, Sandalio 1, Santos 3, Teodorino 1, Tito 1, Trinidad (como nombre primero) 2, Valente 2, Venisio 1 y Venustiano 1.-Se manifiesta cierto gusto por los nombres germánicos: Carlos 18, Enrique 8, Eduardo 7, Federico 6, Conrado 3, Ricardo 3, Romualdo 3, Lamberto 2, Adolfo 1, Arnulfo 1, Gildardo 1.

1890: Hay pocas advocaciones marianas (Guadalupe 8, Luz 8, Concepción 2, Dolores 2, Carmen 1, Encarnación 1, Nieves 1, Soledad 1), y el mismo nombre de Maria no sale más que 27 veces. En cambio están de moda el (Sagrado) Corazón de Jesús 26, el Sagrado Corazón de Maria 3 y la Santisima Trinidad 12. Hasta aparecen los tres en un solo nombre: Pedro José Eulogio Miguel Francisco Javier de los Sagrados Corazones de Jesús y de Maria y de la Santisima Trinidad. Se cita una vez el Nombre de Jesús. La veneración de los santos se manifiesta ya poco: Luis Gonzaga 8, Francisco de Paula 4, Francisco Javier 3, Felipe de Jesús 3, Francisco de Asis 2, Juan Bautista 2, Juan de Mata 2, Pedro de Alcántara 1, Domingo de Guzmán 1, Juan Nepomuceno 1.De nombres femeninos se derivan Brigido 1, Catalino 1, Elso 1, Lauro 2, Magdaleno 6, Margarito 1, Petronilo 1, Rito 2 y Susano 1, e incluso Salomé. Del Viejo Testamento se sacaron Abraham 1, Benjamin 1, Daniel 11 (!), David 1, Efrén 1, Ezequiel 1, Isaac 2 y Moisés 1.-Pero lo que más nos llamó la atención en 1890 es la boga de los nombres de origen germánico: Abelardo 1, Adalberto 2, Adolfo 6, Adelaido 1, Alberto 11, Alfonso 4, Alfredo 5, Anselmo 1, Armando 3, Arnulfo 2, Baldomcro 2, Beltrán 1, Bernardino 1, Bernardo 1, Carlos 11, Dagoberto 1, Edmundo 1, Eduardo 8, Fnrique 15 (!), Frnesto 3, Everardo 1, Federico 3, Fernando 7, Filiberto 1, Gerardo 2, Gonzalo 3, Gualberto 1, Gumersindo 1, Gustavo 2, Heriberto 1, Hermelindo 1, Leonardo 1, Leopoldo 3, Raúl 1, Raymundo 2, Ricardo 5, Roberto 7, Rodolfo 2, Rogelio 1, Romualdo 2, Walter 1, Wilebaldo 1.-Otros nombres poco usuales son César 1, Crisóteles 1, Emigdio 2, Fausto 1, Fidencio 3, Fortunato 1, Galdino 2, Hermilo 1, Honorato 1, Inocente 1, Lioorio 1, Nabor 1, Nicanor 1, Pascasio 1, Proceso 1, Próspero 1, Rutilio 1, Sabino 1, Silverio 1, Sotero 1, Tito 1, Vidal 1, Vito 2, Zacarias 1 y Zenón 2.

Nombres femeninos

Entre 1660 y 1675 goza de enorme popularidad la combinación Maria Theresa, por ser el nombre de la Infanta que se casó en 1660, 
a la edad de 22 años, con Luis XIV de Francia. Así en 1675 ocupa Theresa el quinto lugar entre los nombres más populares (nada menos que 38 veces). Son todavía más populares Gertrudis 54 y Rosa 41.

1760: Nombres religiosos casi todos. Maria se da 282 veces, Josepha 186, Manuela 28, Luz 17, Dolores 14, Guadalupe 10, Loreto 7, Jesús 4, Encarnación 4, Trinidad 4, los Angeles 2, Carmen 2, Rosario 2, Candelaria 1, Cruz 1, Felicitas 1, Perfecta 1, Presentación 1, el Smo. Sacramento 1 y la Sangre de Cristo 1. Paula (San Francisco de) se nombra 5 veces, Nepomuceno 4, Javier 2, Longinos 2. Sendas veces aparecen San Bruno, San Camilo, San Miguel, San Onofre y Piaquinta (!).Entre los nombres poco comunes se destacan América 1, Astacia 1, Agabona 5, Amatista 1, Eudacia 1, Cordiana 1, Crisóstoma 1, Giorgia 2, Leonida 1, Lucinda 1, Narcisa 1, Nicanora 1, Onofria 1, Praxedis 1, Prisca 1, Próspera 1, Roncaba 1, Simeona 1, Rosalina l y Venancia 1.

1779-80: La Sagrada Familia figura en la mayoría de los nombres. Maria aparece 329 veces (¡283 como elemento primero!), Josefa 149 y Jesús 13. El culto de la Virgen se manifiesta además en Guadalupe 33, Dolores 14, Loreto 10, Carmen 8, Luz 7, Concepción 2, Regina 2, Rosario 2, Asunción 1, Encarnación 1, Paz 1, Pilar 1 y Soledad 1.-Aparece tres veces el extraño nombre Cronistas.-Entre los derivados del masculino apuntamos Blasa 1, Casimira 1, Dámasa 1, Daria 1, Diega 1, Fermina 1, Lázara 1, Martina 1, Plutarca 1, Policarpa l y Simona 1.Otros nombres de poca frecuencia son: Arcadia 1, Benigna 1, Cleofas 2, Amata 1, Fulgencia 1, Cleta 1, Epitasia 1, Gabina 1, Evarista 1, Filomena 1, Euthemia 1, Germana 1, Egidia 2, Franciala 2, Cornelia 2, Cándida 1, Gorgonia 2, Demetria 1, Modesta 2, Donaciana 1, Atilana 1, Eduarda 1, Leocadia 1, Irinea 1, Hortensia 1, Gerundia 1, Emeteria 1, Anacleta 1, Lugarda 2, Máxima 1, Hidalia 1, Pia 1, Plácida 1, Nazaria 1, Protasia 2 y Gervasia 3 (San Protasio y San Gervasio fueron hermanos), Romualda 1, Remigia 1, Salomé 1, Urbana 1, Valeriana 1, Verónica 1, Ladislao 1 y Wenceslao 1.

1800: Ha crecido notablemente la boga de los nombres múltiples y de los que expresan culto a los santos y a la Sagrada Familia. Entre los 400 bautismos femeninos que analizamos, figura 327 veces Maria como nombre primero y otras 40 como nombre secundario, lo que quiere decir que en 1800 más del $90 \%$ de todas las niñas mexicanas recibieron ese nombre. Al esposo de la Virgen aluden los 137 Josefa, al mismo Cristo los 37 Jesús y los 22 Manuela, mientras que la Santisima Trinidad se nombra 11 veces, Dios y la Santa Cruz 5 veces cada uno, 2 veces el Santisimo Sacramento y 1 vez el Espiritu Santo.-Contamos además 185 alusiones a misterios de la Virgen. Los más populares son Guadalupe 68, Dolores 44 y Loreto 21. Luego siguen Luz 12, Concepción 8, Carmen 6, Merced 5, Encarnación y Paz y Soledad 3, Angeles y Pilar 2, y Asunción, Expectación y Piedad 1.-Entre los santos, llega a su apogeo la devoción a (Luisa) Gonzaga 45. Son muy populares también (Francisca de) Paula 19 y (Juana) Nepomuceno 17. Con menor frecuencia apuntamos (Francisca) Xaviera 5, (Juana) Evangelista 3, (Francisca de) Borja 2, (Vicenta) Ferrer 1, 
(Francisca de) Asis 1, San Agustin 1, San Marcos 1, Viterba 1 y Vidal 1.Otros nombres bastante comunes en 1800 , aunque no hay lugar para relacionarlos con un solo individuo, son Agustina 41, Joachina 38, Vicenta 26, Rafaela 19, Ramona 16 y Michaela 15.-Derivan de nombres masculinos Daría 2, Diega 3, Dionisia 2, Plutarca 1, Rómula 1.-Como nombres poco comunes apuntamos Maria de Africa 1, Cástula 1, Digna 1, Febronia 2, Guntitiana 1, Praxedis 3, Nemesia 1, Pámfila 3 y Venancia 1.

1820: De 400 niñas bautizadas, 337 recibieron Maria como primer nombre y 23 más como nombre secundario, o sea el $90 \%$.-Nombres derivados del masculino: Aleja 1, Antioca 1, Anselma 3, Apolinaria 1, Bautista 1, Blasa 1, Bonifacia 2, Eduarda 2, Estanislá 2, Evangelista 1, Fabiana 1, Felixa 1, Fermina 2, Fernanda 1, Gervasia 1, Gabriela 4, Guillerma 1, IIiginia 2, Hilaria 1, Hipólita 4, Ignacia 32, Irinea 2, Isidora 1, Jacoba 2, Jacinta 3, Javiera 2, Jerónima 1, Joaquina 11, Josepha 113, Leandra 1, Leona 1, Lorenza 5, Manuela 22, Matea 1, Maximina 2, Nicolasa 5, Norberta 1, Obispa 1, Octaviana 2, Onofra 3, Pantaleona 2, Pascuala 5, Pioquinta 1, Porfiria 1, Procopia 1, Ricarda 1, Rómula 1, Sebastiana 1, Silvestra 1, Simona 5, Sixta 1, Timotea 1, Tomasa 2, Urbana 2, Vicenta 20, Wenceslaa 2.

De las Marias, 220 llevan además nombres que aluden a advocaciones o a misterios de la vida de la Virgen. Así tenemos:

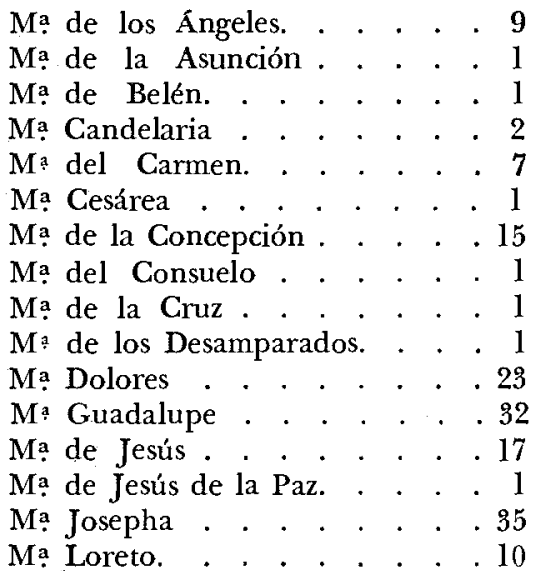

Ma de la Luz. . . . . . . . 15

Ma de la Merced . . . . . . 2

$M$ a de las Nieves . . . . . . 1

Ma de la Paz . . . . . . . 3

Ma Perfecta . . . . . . . . 1

Ma de la Piedad. . . . . . . 1

Ma del Pilar. . . . . . . . 1

Ma del Refugio . . . . . . . 1

Ma Regina. . . . . . . . , 2

Ma Romana . . . . . . . . 1

Ma del Rosario . . . . . . , 2

Ma (de la) Soledad. . . . . 28

Ma (de la) Soledad de la Cruz. 1

Ma (de la) Soledad de Jesús. . 1

Ma (de la) Soledad de S. Marcos. 1

Ma Trinidad. . . . . . . . 3

Tampoco faltan dichos epítetos en otras combinaciones, por ejemplo Juana del Carmen o Josefa del Rosario. Hay repetidas alusiones a los otros miembros de la Sagrada Familia, más advocaciones a varios santos. El nombre de Maria aparece un total de 360 veces, el de Josepha 113, el de Jesús 48, la (Santisima) Trinidad 26, los Angeles 11, los Santos Reyes 3, el (Sagrado) Corazón 2, y una vez la Divina Providencia. Soledad figura un total de 55 veces, Dolores 47, Guadalupe 40, Concepción 23, Luz 21, Loreto 12, Carmen 10, Paz y Regina 5, Cruz y Rosario 4, Pilar 3, Candelaria y Merced 2, y sendas veces Asunción, Belén, Consuelo, Encarnación, Nieves, Piedad y Refugio. 
1869: Todavía hay buen número de advocaciones marianas. Las $\mathbf{5}$ más frecuentes son Guadalupe 41, Concepción 32, Soledad 30, Luz 26 y Dolores 19. De los santos, el único muy citado es Francisco de Paula 13. Hay varios nombres raros, exóticos o inventados: Aida (la ópera de Verdi del mismo nombre se estrenó ese año), Carmelina 1, Coleta 1, Cristiana 1, Elisabel 1, Esmeralda 1, Hernoalda 1, Jocunda 1, Ermina 1, Melinda 1, Melquiades 2, Norma 1, Otilia 1, Pascasia 1. También hay varios derivados de nombres masculinos: Adalberta 1, Alberta 1, Anselma 1, Arcadia 1, Bonifacia 1, Clementa 1, Dámasa 1, Damiana 1, Diega 1, Eduarda 1, Eriberta 1, Enriqueta 1, Ernesta 1, Ernestina 1, Estanisla 2, Eabiana 1, Federica 1, Genara 1, Hermenegilda 1, Isidra 1, Jacoba 3, Jocunda 1, Macaria 2, Narcisa 1, Nestora 3, Pánfila 1, Pascasia 1, Pascuala 1, Reinalda 1, Ricarda 1, Roberta 1, Rodriga 1, Silvestra 1, Venustiana 1.-De nombres hebreos sólo notamos Esther 5, Sara 5 y Réeca 1.

1890: Mientras que en la segunda mitad del siglo xIx las advocaciones de la Virgen van extinguiéndose en la onomástica masculina (sólo 24 en 1890, en 1910 sólo 7, en 1930 cuatro, en 1952 tres), en la femenina aún conservan vitalidad. Contamos en 1890 nada menos que 240 advocaciones: Guadalupe 45, Luz 34, Dolores 27, Carmen y Concepción 25, Soledad 13, Angeles 11, Esperanza y Paz 7, Loreto 5, Altagracia y Asunción 4, Candelaria y Encarnación 3, y otras tantas veces Merced(es), Pilar y Refugio, Adoración 2, Consuelo 2, Lourdes 2, Piedad 2, Rosario 2, Socorro 2, y sendas veces Belén, Consolación, Engracia, Nieves, Remedios y el nombre completo de Virgen de Guadalupe ${ }^{10}$.-Igual que entre los masculinos, se manifiesta en 1890 una piadosa devoción al Sagrado Corazón de Jesús (nombrado 21 veces) y a la Santisima Trinidad (16 veces). Jesús aparece también 14 veces sin el Sagrado Corazón y otras 4 veces en la combinación (Dulce) Nombre de Jesús.-No se acusa entre los nombres femeninos esa boga de los nombres germánicos que caracteriza en 1890 a los masculinos. Hay unos cuantos hebreos (Esther 8, Jacoba 2, Raquel 6, Rebeca 1, Sara 7), algunos derivados de masculinos, por ej. Cira 1, Diega 1, Dionisia 2, Eutiquia 1, Fernanda 1, Gonzala 1, Guillerma 1, Horacia 1, Ildefonsa 1, Isaura 2, Isidra 1, Jacoba 2, Ladislaa 1, Lamberta 1, Matea 2, Narcisa 2, Nicanora 1, Norberta 1, Pascuala 4, Ruperta 1, Severina 1, Simona 1, y algunos otros que citamos por ser raros: Alma 1, Angelina 1, Carolina 1, Celedonia 1, Celerina 1, Eladia 1, Emma 1, Estrella 1, Eudoxia 1, Filiberta 1, Herlinda 2, Hesiquia 1, Juvencia 1, Lilia 1, Liliada 1, Melinda 3, Otilia 1, Serapia 1.

10 Todavía en 1910 hallamos 155 advocaciones marianas entre los nombres de mujer, pero en 1930 se reducen a 118 y en 1952 a sólo 79 . Si la tendencia continúa $\mathrm{y}$ se acelera como las otras que hemos notado en este estudio, se puede pronosticar que para el año 2000 ya habrán desaparecido por completo. 
EL SIGLO XX

Creemos percibir en la onomástica mexicana del siglo xx una progresiva disminución, más rápida en la masculina que en la femenina, del fervor religioso que movía a los padres de recién nacidos en siglos anteriores. Algunos nombres femeninos sí lo manifiestan todavía, pero son cada vez más numerosos los que parecen haberse escogido, no por razones religiosas, sino simplemente por la eufonía, el gusto por lo exótico u original, o por hacer honor a un célebre artista o figura política. Poco a poco, la onomástica mexicana se va secularizando.

La Revolución mexicana parece haber dado cierto impulso a las tendencias que ya se vislumbran en las postrimerías del siglo pasado, pero no logramos atribuirle ningún cambio decisivo. Tampoco parece haber repercutido en la onomástica de la capital del país, por lo menos no estadísticamente, el notable esfuerzo oficial por idealizar en México la historia y la cultura precolombinas. Entre los miles de nombres analizados no encontramos ni un solo azteca.

Nombres femeninos

En 1910, los nombres de pila femeninos conservan todavía el fervor religioso que ya va desapareciendo de los masculinos, pero éste se limita ahora a nombrar a la Virgen. Maria aparece un total de 240 veces (227 veces como elemento inicial), Guadalupe 33 veces, Luz 24, Carmen 23, Dolores 14, Concepción 12, Consuelo 9, Angeles 8, Esperanza 7, Asunción 6, Candelaria, Encarnación y Soledad 5 cada uno, Refugio(s) y Rosario 4. Otras advocaciones son Mercedes, Nieves, Purificación, Carmelo, Socorro, Gloria, Belén, Cruz, Paz, Felicidad, también Felicitas y Maria Felis (sic), Librada, Perfecta, Virginia, Santisima, y del Sagrado Corazón de Jesús. Algunos nombres presentan concatenaciones como Maria de la Luz Carmen del Sagrado Corazón. Una vez aparece Elisa Fe del Dulce Nombre de Jesús. Pero, salvo dos alusiones a Sta. Teresa de los Angeles, parecen haber desaparecido los nombres de santos de ambos sexos. En cuanto a la Sagrada Familia, Josefa se da sólo dos veces y Josefina seis.

1932: Josefa y Mariana han desaparecido totalmente. Maria se da 194 veces (160 como nombre inicial), y además contamos 123 advocaciones de la Virgen: Carmen 26, Guadalupe 16, Consuelo 10, Concepción 8, Angeles, Esperanza y Luz 7, Rosario y Socorro 6, Dolores 5, Felicitas, Mercedes, Pilar, Refugio y Soledad 3, Asunción y Lourdes 2, Altagracia, Amparo, Aurea, Candelaria, Loreto y Purificación 1. Salvo una sola aparición de San Francisco de Paula, ya no se nombra santo alguno. Jesús se nombra 9 veces, el Niño Jesús 3, el Sagrado Corazón 3 y una vez la Santa Cruz.-A medida que va perdiendo terreno el ele- 
mento religioso en los nombres de pila, se reduce perceptiblemente la proporción de los nombres múltiples. La nueva tendencia parece pronosticar una vuelta al gusto por el tipo de nombre sencillo que predominaba en el siglo xvi. Pero mientras que el siglo xvi se contentaba con un inventario de nombres relativamente limitado, el siglo $\mathrm{xx}$ busca la variedad. Entre nombres exóticos como Abdulia 1, Alma 1, América 1, Amira 1, Artemisa 1, Elia 1, Elsa 2, Elva 1, Elisa 1, Elpidia 1, Mirella 2, Norma 1, Olga 3, Sofía 2, Ursina 1, Zaide 1, Zoila 2 y Zoraida 1, notamos algunos ingleses (Edith 1, Nelly 1) y hebreos (Esther, Judith 2, Raquel 1, Rebeca 5, Sara 3). Están muy de moda los nombres que terminan en -ina y -elia (o -ilia): Albertina 1, Alejandrina 2, Angelina 6, Avelina 1, Carolina 1, Catalina 1, Celina 2, Clementina 1, Delfina 1, Ernestina 1, Evangelina 2, Georgina 2, Joaquina 1, Josefina 10, Justina 1, Ursina 1, Amelia 5, Aurelia 1, Cclia 9, Delia 1, Emilia 1, Eulalia 2, Evclia 5, Fidelia 1, Gudelia 1, Lilia 3, Natalia 2, Ofelia 3. Con el sufijo -eta aparecen Antonieta 3, Enriqueta 5 y Julieta 1.

1952: Ya no se manifiesta en los nombres de pila la devoción a los santos. Josefa, de 1700 a 1830 el nombre más usado después de Maria, queda casi olvidado. (Aparece una sola vez.) Maria sigue todavía popular: 175 (156 veces como elemento inicial), pero solamente contamos un total de 90 advocaciones, entre las cuales se destacan Guadalupe 24, Carmen 12, Lourdes 11, Luz 8, Concepción 5.-En cambio, ha crecido mucho el número de nombres nuevos y exóticos. Pero éstos ya no suelen ser múltiples como en siglos pasados. Unas veces se desentierran del santoral los nombres de santos casi olvidados, o se inventan nombres o variantes de ellos (como Daria 2, Elia 2, Estéfana 1, Romina 1, Rosela 1, Yadira 1); otras veces se toman del hebreo o del inglés ( $\mathrm{Ju}$ dith 2, Noemi 1, Raquel 1, Rebeca 2, Ruth 1, Sara 4, Elizabeth 1, Pamela 1).

Nombres masculinos

1910: Hay un número creciente de nombres hebreos (Benjamin, Daniel, David, Efrain, Jacobo, Rubén, Samuel) y paganos (Alejandro, Anibal, César, Augusto, Dario, Héctor, Horacio, Plinio, Ulises).-Pero lo que más se destaca es la enorme popularidad de nombres de origen germánico. Hay por ejemplo 24 Enrique $(13+11)$, 18 Carlos $(12+6)$, 16 Alfonso $(7+9), 15$ Francisco $(7+8), 14$ Fernando $(8+6), 13$ Eduardo $(9+4), 12$ Guillermo $(7+5), 10$ Jorge $(9+1)$, y 9 Alberto $(2+7)$ -todos ellos nombres dinásticos de las casas reales europeas de la época-, más varios Adolfo, Alfredo, Armando, Arnoldo, Bernardo, Ernesto, Everardo, Federico, Gerardo, Gilberto, Gustayo, Humberto, Olaf, Raimundo, Ramón, Raúl, Ricardo, Roberto, Rodolfo y Waldo.

Entre la primera y la segunda guerra mundial se acrecienta la boga de los nombres germánicos. Así lo prueban en 1932 Abelardo 1, Adalberto 3, Adolfo 1, Alberto 9, Alfonso 13, Alfredo 6, Alvaro 1, Anselmo 1, Armando 9, Arnulfo 2, Bernardo 1, Carlos 17, Dagoberto 1, Edmundo 1, Eduardo 7, Elfego 1, Enrique 14, Ernesto 4, Federico 1, 
Nombres masculinos

$\begin{array}{rr}1910 & 1930 \\ 400 & 400 \\ 269 & 288 \\ 187 & 173 \\ 153 & 193 \\ 60 & 34 \\ 170 & 156\end{array}$

Cuapro IV: 1910-1952

1952
400
228
218
178
4
143

Nombres femeninos

$\begin{array}{rc}1930 & 1952 \\ 400 & 400 \\ 275 & 256 \\ 110 & 128 \\ 211 & 245 \\ 79 & 27 \\ 174 & 158\end{array}$

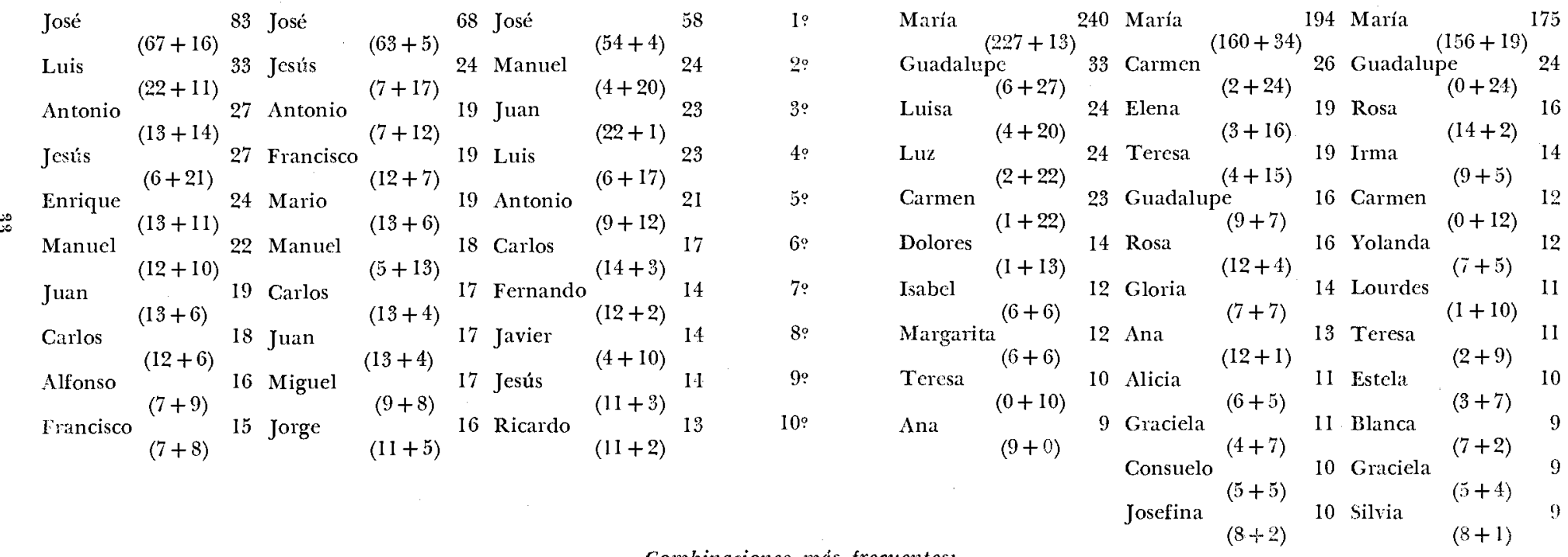

\begin{abstract}
15
7
6
5
5
\end{abstract}

$\begin{array}{cr} & 1910 \\ \text { Nombres analizados } & 400 \\ \text { Nombres diferentes } & 236 \\ \text { Nombres sencillos } & 118 \\ \text { Nombres dobles } & 202 \\ \text { Nombres múltiples } & 80 \\ \text { Elem. diferentes } & 120\end{array}$

10
180
18
80
120

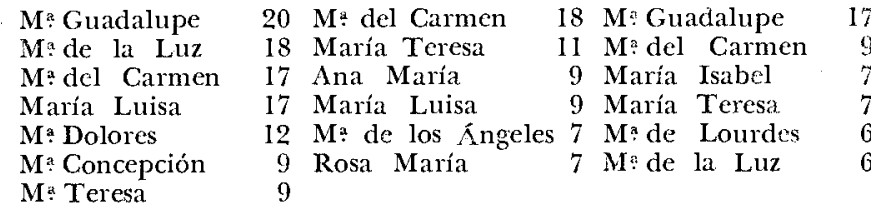


Fernando 9, Francisco 19, Gerardo 2, Gilberto 3, Gonzalo 2, Guillermo 6, Gumaro 1, Gumersindo 2, Gustavo 5, Henri (!) 1, Heriberto 3, Humberto 6 (germánico, aunque en 1932 debe su popularidad sin duda al Príncipe Humberto de Italia), Leobardo 1, Leonardo 6, Leopoldo 1, Leovigildo 1, Luis 14, Norberto 1, Oscar 4, Raimundo 2, Ramón 6, Raúl 13, Ricardo 2, Roberto 10, Rodolfo 4, Rodrigo 1, Roge. lio 1.-Los nombres hebreos del Viejo Testamento están representados

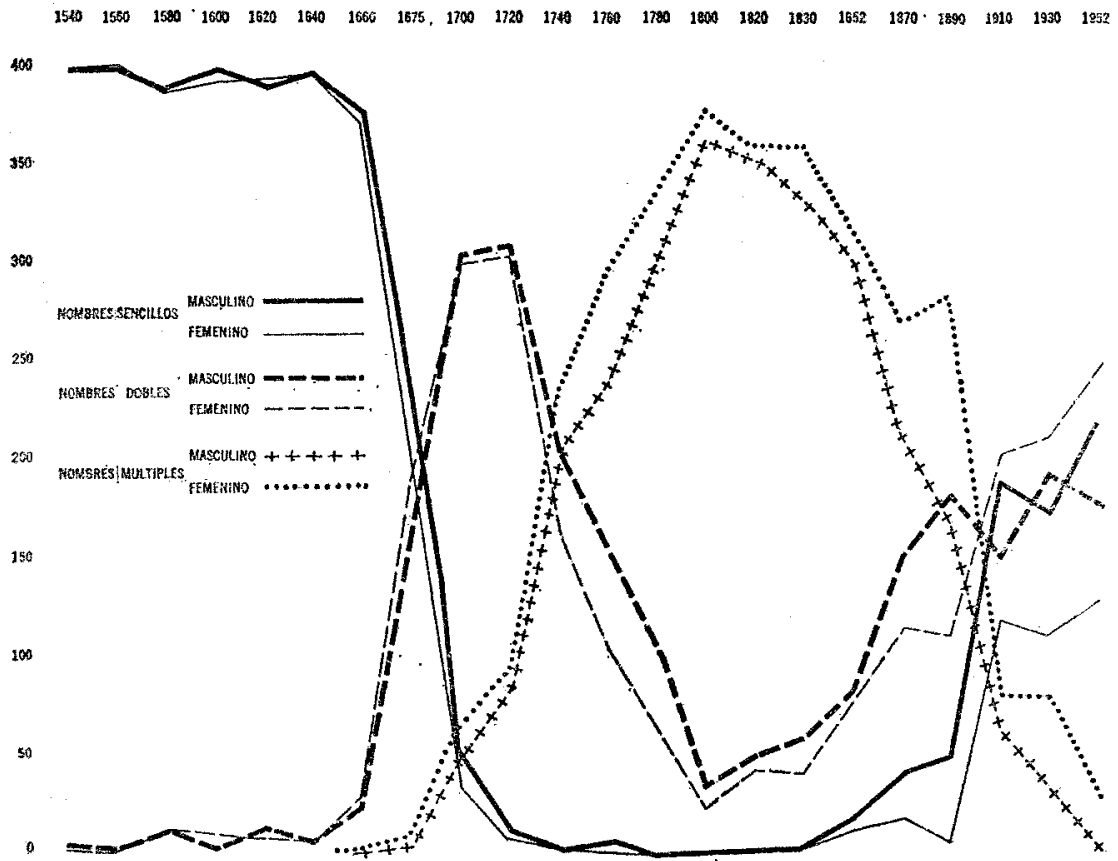

por Abraham 2, Benjamin 2, Daniel 4, David 3, Efrén 1, Isaac 1, Isaías 1, Jacobo 1, Moisés 3, Ruóén 5, Samuel 2.-En cambio, los elementos de carácter claramente religioso casi han desaparecido de la onomástica masculina. Se da 24 veces Jesús ( 7 veces como inicial), pero Maria ha desaparecido por completo. Solamente encontramos Carmen 1, Guadalupe 1, Remedios 1, Rosario 1, Corazón de Maria 1, Sta. Teresita del Niño de Jesús 1, Smo. Sacramento 1, Sagrado Corazón 1, Juan de Dios 2 y Trinidad 3.-Los dos Victor Manuel que aparecen en 1932 se deben quizás al nombre del rey de Italia Víctor Manuel III, así como los 6 Humberto reflejan el de su hijo.

1952: Las mismas tendencias se dejan ver en la onomástica masculina de 1952, según se verá por el análisis completo que ofrecemos en el apéndice IV, y por el cuadro estadístico y el gráfico que aparecen en esta página y en la anterior. 


\section{RESUMEN Y CONCLUSIONES}

En este estudio cronológico-estadístico de los nombres de pila mexicanos hemos podido indentificar tres etapas principales:

1) La primera (1540-1660) se caracteriza por los nombres sencillos y poco variados para ambos sexos.

2) En la segunda (1675-1890), el fervor religioso se manifiesta a cada paso en el gusto por los nombres largos y llenos de advocaciones a la Virgen, a diversos santos, y a la Sagrada Familia.

3) En la actual (1910-?), seguramente de transición, el tono religioso va cediendo a un gusto netamente secular por lo exótico $\mathrm{u}$ original (nombres germánicos, hebreos, mitológicos o inventados), por los nombres de personajes contemporáneos de fama mundial (como Eduardo, Alfonso, Humberto y Victor Manuel), o por la mera eufonía. La variedad de nombres es realmente extraordinaria, aun sin tomar en cuenta la creciente cantidad de formas afectivas a que suelen dar lugar ${ }^{11}$.

En cuanto al número de elementos que suelen tener los nombres mexicanos de la actualidad, el tipo más común es ahora, para las niñas, el de dos elementos, y para los varones, el sencillo, como en el siglo xvI.

Para concluir, hemos hecho constar que a pesar de ser cada bautismo un fenómeno único, y la selección de los nombres de pila al parecer el producto de la libre voluntad de los padres del recién nacido, en su conjunto dichas selecciones quedan sometidas, en cada momento, al tiránico gusto colectivo de la época.

State University of New York at Buffalo.

Peter Boyd-Bowman

\section{APÉNDICE I $a: \quad 1540-1545$}

400 nombres de pila masculinos

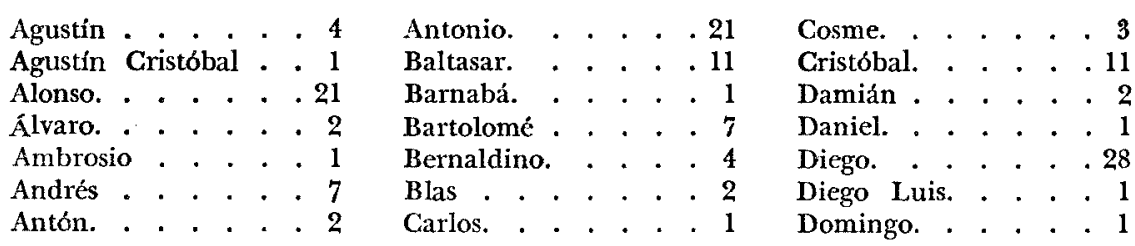

11 Consúltese sobre el particular Peter Boyd-Bowman "Cómo obra la fonética infantil en la formación de los hipocorísticos", NRFH, 9 (1955), 337-366, donde recogimos y clasificamos centenares de formas afectivas usadas en Hispanoamérica e indicamos para cada una su distribución geográfica. 
Enrique . . . . . . 2

Esteban . . . . . 3

Feliciano. . . . . . 1

Felipe. . . . . . 3

Fernando. . . . . . 4

Francisco. . . . . 28

Gabriel . . . . . 1

García. . . . . . 4

Gaspar .. . . . . 11

Germán. . . . . . 1

Gonzalo. . . . . 4

Gregorio. . . . . 1

Hermán. . . . . , 1

Hernando . . . . . 11

Hipólito . . . . . . 1

Jerónimo . . . . . . 5
Jorge . . . . . . . 1

$\operatorname{Jose}(\mathrm{pl})$. . . . . 3

Juan . . . . . . . 79

Juan Luis. . . . . I

Julián . • . . . . . 1

Lázaro . . . . . . . 1

Leonardo. , . . . . 1

Lope . . . . . , 2

Lorenzo . . . . . . 1

Lucas. . . . . . . 2

Luis . . . . . . . 20

Manuel . . . . . 3

Marcos . . . . . . 1

Martín. . . . . . 6

Mateo. . . . . . 2
Matías. • . - . . . 1

Melchior . . . . . 11

Miguel . . . . . . 5

Nicolás , . . . . 4

Nuflo . . . . . . . 1

Pablo. . . . . . 2

Pedro. . . . . . 29

Rodrigo . . . . . . 7

Ruy . . . . . . . 1

Sancho. . . . . . . I

Sebastián , . . , 1

Serván. . . . . . 2

Silvestre . . . . . 1

Tomás. . . . . . 2

Victoriano . . . . . 1

AlÉNDICE I $b: 1540-1545$

100 nombres de pila femeninos

Agustina . . . . . 2

Aldonza . . . . . 2

Ana . . . . . . 47

Anastasia. . . . . 1

Andrea . . . . . 4

Angela . . . . . . 1

Antona . . . . . 1

Antonia . . . . . 3

Beatriz. . . . . 26

Bernaldina . . . . 1

Bernardina. . . . . 1

Casilda . . . . . . 1

Catalina . . . . . 28

Clara . . . . . . 1

Costanza .. . . . . 3

Crispiniana. . . . . 1
Engracia . . . . . . 1

Felipa . . . . . 1

Francisca. . . . . 18

Guiomar. . . . . 1

Ines . . . . . . 15

Isabel. . . . . . . 52

Jacoba. . . . . . 1

Jerónima. . . . . . 12

Juana . . . . . . 23

Juliana . . . . . . 4

Leonor . . . . . . 24

Lucía . . . . . 1

Luisa . . . . . .

Luisa Agustina . . . 1

Ma(g)dalena . . . . 7

Margarita . . . . 4
María. . . . . . 69

María Magdalena, . 1

Mariana. . . . . . 5

Marina . . . , , 1

Marta. . . . . . 1

Melchiora , . . . 4

Mencía . . . . . . 1

Nicolasa. . . . . . 3

Pascuala. . . . . , 1

Petronila. . . . . 2

Polenciana . . . . , I

Polonia . . . . . . 1

Sebastiana . . . . . 1

Simona . . . . 1

Ursula . . . . . . 4

Violante. . . . . 1

ApÉNDICE II $a: 1660-1661$

400 nombres de pila masculinos

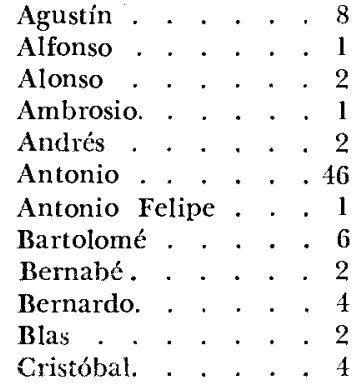

Diego. . . . . . . 14

Diego Javier . . . 1

Diego Martín . . . . 1

Domingo. . . . . . 4

Esteban . . . . . . 3

Felipe. . . . . . . 11

Francisco. . . . . . 23

Francisco Javier . . . 1

Fulgencio . . . . . 1

Gabriel . . . . . 5

Gregorio. . . . . . 4

Hipólito, . . . . . 2
Ignacio . . . . . . 5

Isidoro. . . . . . . 1

Isidro Vicente. . . . 1

Jacinto . . . . . . 2

Jerónimo. . . . . . 1

Joaquín. . . . . . 2

Joseph (e) . . . . . . 62

Joseph Gervasio . . . 1

Juan . . . . . . . 63

Juan Antonio. . . . 4

Juan Blas . . ... 1

Juan Eligio . . . I 
Juan Félix . . . . 1 Juan Francisco. . . . 2 Juan Ignacio . . . . 1 Juan Jorge. . . . . 1 Juan Miguel . . . . 1 Laureano Alfonso. . 1

Lorenzo . . . . . . .

Lucas ... . . . . .

Luis . . . 3

Manucl . . . . . 14
Manuel Francisco . . 1

Marcos . . . . . . 2

Mateo. . . . . . 2

Matías. . . . . . 2

Michacl . . . . . . 1

Miguel . . . . . . 10

Miguel Antonio, . . 1

Miguel Cristobbal. . . 1

Nicolás . . . . . 16

Onofre . . . . . . 1

APÉNDICE II $b: 1660-1661$

400 nombres de pila femeninos

Agueda . . . . . 1

Agustina. . . . . . 3

Ana. . . . . . . . 11

Ana María. . . . . 1

Andrea . . . . . . 1

Angela. . . . . . . I

Antonia . . . . . 41

Antonia Luisa . . . 1

Baltasara. . . . . . 1

Bárbola . . . . . . 1

Beatriz . . . . . 1

Bernabela . . . . . 1

Bernarda. . . . . . 1

Blasia. . . . . . 1

Catalina .... . 10

Catalina María . . I

Catherina .... . 1

Cecilia . . . . . 1

Clara . . . . . . 3

Damiana .. . . . . 1

Dominga. . . . . . 1

Dorotea Antonia. . . 1

Elugarda. . . . . . 1

Felipa. . . . . . 3

Francisca . . . . 17

Francisca María . . 1

Gertrudis. . . . . 7
Getrudis, . . . . . 6

Gregoria . . . . . . 1

Hipólita. . . . . . 1

Ignacia Josefa. . . . 1

Inés

Inés María. . . . . 1

Isabcl. . . . . . . \$

Isabel Antonia . . . 1

Jacinta . . . . . 1

Jerónima. . . . . . 3

Joaquina . . . . . . 1

Josefa. . . . . . . 91

Josefa María. . . 2

Juana. . . . . . . 39

Juana Francisca . . . 1

Juana Getrudis . . . 1

Juana María. . . . 3

Juana María Teresa . 1

Leonor . . . . . . 4

Lorenza . . . . . . 2

Lucía . . . . . . . 1

Luisa . . . . . . 4

Ma (g) dalena . . . . 3

Manucla. . . . . 8

Marcela . . . . . . 1

Margarita. . . . . 1
Pascual . . . . 2

Pedro. . . . . . 12

Ramón . . . . . . 1

Rodrigo . . . . . . 1

Roque. . . . . . . 2

Salvador ... . . . 6

Sebastián. . . . . . 5

Sebastián Juan . . . 1

Tomás . . . . . 11
María. . . . . .93

Maria Antonia . . . 1

María Bernarda . . . 1

María Felipa. . . . 1

María Josefa . . . . 1

María Magdalena . . 1

María Manuela . . . 1

María Micaela . . . 1

María Nicolasa . . . I

María Teresa . . . . 1

María Victoria . . I

Mariana . . . . . 3

Mariana Francisca. . 1

Melchiora . . . . . 1

Micaela . . . . . 11

Nicolasa . . . . . 5

Nicolasa Francisca. - 1

Pascuala. . . . . . 1

Petrona . . . . . . 3

Petronila. . . . . . 2

Polonia . . . . . . 1

Sebastiana . . . . . 2

Teodora . . . . . 1

Teresa. . . . . . 10

Teresa María . . . . 1

Tomasa . . . . . 5

Ursula. . . . . . . 2

ApÉndice HI $a: 1852$

Nombres masculinos

Abundio Pío

Agapito Mateo José $\mathbf{M}^{a}$ de Jesús

Agustín Francisco

Agustín Fro de Paula Cesario

Agustín Ignacio Manuel de Sta. Teresa

Agustín Juan

Agustín Lorenzo
Agustín María

Agustín Ma Cesario Jesús José de la Sma. Trinidad

Alejandro Fro de Paula del Corazón de Jesús

Ambrosio Antonio

Andrés Avelino Martín 
Angel del Rosario

Antonio*

Antonio del Carmen

Antonio Felipe Neri Cleofas Miguel

Antonio Ma Regino Agustín Nemesio

Juan Nepomuceno Ignacio

Antonio de Padua Julio

Apolonio Legonio Pedro de Jesús

Arcadio Higinio

Aurelio Amado José

Benigno Catarino

Benigno Catarino Valente

Bernardo Maximiano de la Soledad

Blas Andrés Corino

Calixto Fortunato

Calixto del Pilar

Camilo Fro Ma de la Soledad

Candelario Blas

Carlos Adolfo Maximiliano Angel

Carlos Alberto Regino

Carlos Ant? de Padua Enrique José Trinidad

Carlos Enrique

Carlos Guillermo Manuel

Carlos Lauro Luis

Carlos Modesto de la Sma, Trinidad

Carlos Simón Gabino Guadalupe

Carlos Simón de la Soledad

Cenobio Claudio Luciano

Cesario Eduardo Emilio

Cipriano Cornelio

Cipriano Miguel

Ciro Félix Luis Gonzaga

Clemente Cecilio Vicente

Cleofas Aurelio

Crisanto Trinidad Simón

Delfino de Jesús

Demetrio Fabiano

Diego

Dionisio Aurelio del Corazón de Jesús

Dionisio Salvador de la Sma. Trinidad

Domingo Juliano Pastor

Eduardo Gil de los Remedios

Eligio Natalio Agustín

Eligio Natalio Concepción Dolores

Emilio Clemente José Pedro Federico Domingo Juan Nepomuceno Estanislao Pascual Ramón Juan Bautista Juan de la Cruz de Gonzaga de Jesús

Enrique Julio Cristóbal

Enrique Vicente Teófilo

Estanislao Homobono

Esteban Marino

Eudivio Adelagdo Alvino José Espiridión

Eugenio Fro de Paula José Manuel

Eugenio Maclovio
Eugenio Maclovio Luis Gonzaga

Evaristo Luis

Federico de Jesús Ignacio

Feliciano Juan Rafael

Felipe de Jesús Fro de Paula Fro Javier Fro Jerónimo Federico Amado Aurelio Anto de Padua de la Trinidad

Félix Abundio

Félix Anto Joaquín Pascual

Femato José Darío

Fernando Gonzalo

Filomeno María

Francisco Ignacio

Fro Jacinto

Fro Javier José Ma de Jesús Rafael Fro de Paula Diego Anto de Padua

Fro de Jesús Ant?

Fro Juan Bautista

Fro Martín Desiderio

Fro de Paula Dámaso Guadalupe Lucio Fr:

Fro de Paula Jesús Anastasio

Fro de Paula José Mariano

Fro de Paula Nativo Librado Rafael

Fro de Paula Tirso Valerio Ignacio de la Sma. Trinidad

Fro Remigio Rosendo

Fructuoso Inés

Genaro

Genaro Fro Javier de Jesús

Gorgonio Fabiano Nicolás Alberto

Guillermo

Guillermo Guadalupe Anto

Gumersindo Clariso Guillermo Ant \% Abad de Jesús

Hilario Jorge Romualdo Manuel

Ignacio

Ignacio de los Angeles Ma Fro de Paula Ramón

Ignacio Martiniano

Jerónimo Honorio Fro

Jesús Camilo Primo

Jesús José M* Luis Miguel Guillermo

Jesús Tomás

Joaquín

Joaquín Ma Mario José

Joaquín Narciso Fro

José Agapito Fro

José Agustín de Santa Bárbara

José Agustín de Santa Rosa

José Ambrosio Pedro Luis Gonzaga

José Anastasio Vicente

José Andrés Alejandro

José Andrés Angel Benito Agustín

José Antonio

José Anto Ignacio Agustín Juan Amado Félix

* Los nombres con asterisco aparecen dos veces; los demás, una solu. 
José Anto Ma Carlos Telesforo Fro de Paula

José Ant? Policarpo

José Asunción Arnulfo Roque Jacinto

Wenceslao

José Aurelio

José Aurelio Mauro

José Bárbaro Melesio Anto

José Bernardo

José Brígido Fro de Jesús

José Bruno Simón Anto

José Calixto

José Camilo Enrique Rafael

José Camilo Pascual

José Camilo de la Soledad

José Carlos Luis Gonzaga

José Casildo Florentino

José Cayetano Anto de la Sma. Trinidad

José Cayetano de Jesús

José Cecilio Emilio

José Cereantomo

José Cesario Agustín Fro de Santa Rosa

José Cirilo Filemón Fro Trinidad

José de la Cruz Crescencio

José Daniel

José Eduardo

José Eduardo Calixto Fortunato

José Eleuterio Manuel

José Eligio Francisco

José Eligio Fro Bibiano

José Eligio Fro Javier

José Enrique Camilo Ignacio Soledad

José Enrique de Jesús Ma Buenaventura

José Esteban Sandalio Miguel

José Eulalio Ant?

José Eulalio Benigno

José Fabiano Sebastián de Jesús

José Federico Cristóbal Ignacio López de San Fro de Paula

José Felipe Jesús Anto Joaquín Ma Agustín

José Filomeno Aristero

José Florentino Lucas

José Fortino Hipólito

José Fro Atilano Bruno Ignacio

José Fr: de Borja

José Fro de Rafael de Jesús

José Fro de la Soledad*

José Gregorio

José Guadalupe

José Guadalupe Agustín Conrado

José Guadalupe Ángel Fr?

José Guadalupe Benisio

José Guadalupe Dámaso

José Guadalupe Fro de la Trinidad

José Guadalupe Hilarión

José Guadalupe Luis Miguel

José Guadalupe Nicolás

José Guillermo Luis Gonzaga
José Gumersindo Hermilio

José Higinio

José Hilario Carlos $M^{\sharp}$ Luciano

José Hilario Luis Rafael

José Ignacio

José Ignacio Catalino

José Ignacio Clemente Juan de la Cruz.

José Ignacio Herculano

José Ignacio de Jesús*

José Ignacio de Jesús Luis Álvaro

José Ignacio Manuel

José Ildefonso Guadalupe Ant: Salvador

José Jacinto Guadalupe $M^{a}$ de la Soledad

José Jacinto Joaquín

José de Jesús

José de Jesús Agapito Agustín

José de Jesús Gonzaga Enrique

José de Jesús Guadalupe Arcadio

José de Jesús Lino Agustín de la Sma.

Trinidad

José de Jesús Manuel

José de Jesús Marcelo

José de Jesús Rufino

José de Jesús de la Soledad

José Joaquín Fro de Paula

José de Juan Miguel

José Juan Nepomuceno de Jesús y M?

Joaquín Bernardo Maximiano Inocen-

cio Lugardo Federico

José Julio*

José Julio Próspero

José Leopoldo Inocencio

José Lorenzo

José Lucas Feliciano de la Sma. Trinidad

José Lucerencio Eugenio

José Luciano Juliano

José Luis Bartolo Federico Ignacio del Corazón de Jesús

José Luis Felo

José Luis Ildefonso de Jesús Joaquín

José Magdaleno Agustín Ma de los Angeles

José Manuel Ant:

José Manuel Cenobio Rafael

José Manuel Rufino

José Manuel Severiano

José Manuel de la Soledad

José María

José $M^{a}$ Ant? Enrique de la Sma. Trinidad

José Ma Carlos Juan de la Cruz Basilio Santiago

José Ma del Carmen Ventura

José Ma Donaciano

José Ma Eduardo del Pilar

José Ma Fro de Asís de la Sma. Trinidad José Ma Gil Constancio Eduardo 
José M: Guadalupe Eniliano

José Ma de Jesús*

José Ma de Jesús Anto de Padua

José M: de Jesús Carlos Mariano Cayctano

José Ma de Jesús Felipe Luis

José M: de Jesús Juan Capistrano Ig. nacio Juan Nepomuceno Agustín Manucl

José Ma Lino

José Ma Luis

José Mạ Luz

José Ma Manucl de la Trinidad Félix de Valusio

José Ma de la Mierced Soledad

José Ma Nicolás de la Preciosa Sangre

José Ma Próspero

José Ma Rafael Juan Nepomuceno Ignacio Clemente

José Ma Ramón Simón Dionisio de Sta. Teresa

José Ma del Rosario

José Má Severo de Jesús

José $M^{a}$ Soledad

José Ma Tirso Fro

José Mariano*

José Mariano Jesús Agustín de Sta. Teresa

José Matías Matco

José de la Merced

José Mcrced Cleofas

José Miguel Agustin Luis Gonzaga

Fabián Sebastián Fro de Paula

José Miguel Gumersindo

José Miguel de Jesús

José Nicolás Dionisio Mariano

José Pedro Alcántara Miguel Lucas

Concepción Feliciano Ant ${ }^{\circ}$ de Jesús

José Paz Timoteo Agustin Policarpo

José Policarpo Paulo Juan Crisóstomo

José Próspero

José Remigio

José Reyes

José Rodrigo Matilde

José Rosa Ma

José Santiago

José Santiago de Jesús

José Santos

José Sebastián Hipólito

José Silvestre Martiniano

José Teófilo Fro de Paula

José Tereso de Jesús Hilarión

José Tiburcio Fortino

José Tiburcio Juan

José de la Trinidad

José Trinidad de los Angeles

José Trinidad Guadalupe Lázaro

José Trinidad Ignacio Conrado

José Vicente Anastasio
José Vicente Ferrer Gumersindo

José Victoriano Ant?

Juan Bautista Maximiliano

Juan Capistrano Santos

Juan Crisóstomo

Juan de la Cruz Cecilio

Juan de la Cruz Conrado

Juan de la Cruz Fro

Juan de la Cruz Gregorio

Juan Esteban

Juan Fro Lauro

Juan Inocencio

Juan José

Juan José Inocencio

Juan Pablo Mario

Juan Pablo Trinidad

Juan Santiago

Julián

Julio José Ma Gumersindo Antonio Ignacio

Justo Cipriano

Lamberto Pedro Colombo de la Sma. Trinidad

Lauro Luis de Jesús

Lázaro Teófilo Luis Gonzaga Agustín

Leonardo Félix Carlos

Leonildes Emiliano

Lino

Lorenzo Tiburcio

Lucio Clemente

Luis

Luis Agustín*

Luis Espiridión de Jesús Gión

Luis Gonzaga Ma Gregorio Fro de Paula Rafael Teresa de la Cruz

Luis Ignacio

Luis Ma Juan Nepomuceno Fro de Paula de la Sma. Trinidad

Luis de Santa Marina

Luis Santiago Eduardo

Luis de la Soledad

Luis Victorio

Macario Ma de la Soledad

Manuel

Manuel de los Ángeles

Manuel Domingo*

Manuel Fabián Sebastián

Manuel Felipe de Jesús Estanislao

Manuel Felipe de Jesús Teófilo Doroteo

Manuel Gildardo

Manuel Gregorio de la Trinidad

Manuel Lázaro

Manuel Ma Apolonio Fro de Paula Santiago

Manuel Ma Jacinto Nicolás Pascual Fro de Paula Luis Gonzaga Juan Nepomuceno

Manuel Ma Pascual Joaquin Juan 
Manuel $M^{a}$ Teófilo Apolinario Vicente de Pablo

Manuel M: Vicente de la Sma. Trinidad Manuel Nicanor Juan Nepomuceno Manuel Pascual

Manuel Prisciliano

Manuel de los Santos Vicente de Paula

Manuel Simón

Manuel Trinidad

Manuel Wenceslao Josć

Marcos Sergio Trinidad

María de Guadalupe Melquíades Dámaso

Ma de la Purificación Miguel

Mariano*

Mariano de Jesús Apolonio

Mario Guadalupe Ignacio de Jesús

Martín

Martín Agustín

Martiniano Macario de Jesús

Miguel Ignacio Juan Crisóstomo

Miguel de Jesús Ant?

Miguel José Eufemio

Miguel Vicente Ramón

Modesto Juan Crisóstomo

Nicolás Ambrosio

Pablo

Pablo Juan Nepomuceno $\mathbf{M}^{a}$ del Pilar

Pedro Alcántara

Pedro Alcántara Miguel

Pedro Andrés Carino

Pedro Pascual Rafael Ant?

Pedro de Santa Bárbara

Pío Amado Vicente Justo

Pío Carlos Augusto Máximo Ant?

Plácido Nicasio Camero Ant? Luis Gonzaga

Porfirio Lamberto de la Sma. Trini. dad de Sta. Rita
Porfirio Loreto de las Cinco Llagas

Pronto Jacinto Cristóbal de Jesús Ma Próspero Julio

Rafael Ricardo Nicasio Feliciano

Ramón Manuel de la Cruz Lorenzo Alberto

Ramón Narciso Salvador

Remigio Fre de Paula Flavio

Ricardo Policarpo Juan Crisóstomo

Rito Simeón Marcial

Romualdo

Romualdo Ma de Jesús

Rosario Cándido Genaro

Roselino Blas Celestino

Sabad Nicolás Guadalupe

Sabrino Venustiano Fro de Paula Leopoldo

Santiago Cristóbal Teodorino

Santiago Evaristo Luis Mario

Santos Carlos

Sebastián José Ricardo

Severo Ignacio Blas

Simón Gabino

Teodoro Fr: de Paula

Tiburcio Modesto Pablo Vicente

Tito Telesforo

Tomás

Tomás Concepción

Trinidad Guadalupe Andrés

Trinidad Tomás Antonio de lit Soledad

Valente Carlos

Vicente Anto Josć Zacarías

Vicente Cristóbal Matías Cecilio

Vicente Justo Rufino Salvador

Viviano Eduardo Santiago Víctor

Wenceslao Miguel Agustín

Wenceslao Miguel Manuel María de la Luz

\section{ApÉNDICG III $b: 1852$}

Nombres femeninas

Adela Guadalupe Petronila Ignacia Adela Josefa Brigida Emetira [¿Emćrita:] Agustina Cesaria

Amada

Amalia $M^{\text {a }}$

Ana Ma Dolores Letrada de la Sma. Trinidad

Ana Ma Manuela Guadalupe Ant?

Andrea Fr. de Paula

Andrea Josefa Aurelia

Ángela

Ángela Ant: Josefa Soledad del Corazón de Jesús
Ángela Matilde de Jesús Concepción

Ángela Remigia Concepción

Atilana Cristina

Aurelia de los Ángeles

Aurelia Natalia

Aurelia Trinidad Guadalupe Fra $\mathbf{M i}$ caela

Bárbara

Belina Rafaela

Bernarda Concepción Guadalupe

Bruna Máxima

Cecilia

Cecilia Remigia Agustina 
Cleta Guadalupe Anta de Padua Concepción Melquíades

Crescencia de la Santa Cruz

Diega Martina Ma Guadalupe Aurelia

Dolores

Dolores Cornelia Amalia

Eduarda Ma del Pilar Faustina Josefa

Elegria Ma de la Soledad

Elena Clara Leonor

Elena Juana de las Nieves Leonidia de los Dolores

Emelia Ma

Eugenia Gertrudis del Pilar

Eugenia Juana Evangelista

Evarista Rafaela Flora de la Purísima Concepción

Faustina Ant del Aguila

Faustina Teresa de la Sma. Trinidad

Felicitas Margarita Amalia

Felipa Francisca de Paula

Felipa de Jesús Polonia

Francisca Atilana

Francisca de Paula

Francisca de Paula Librada Rafaela

Gregoria de Jesús Fra de Paula

Guadalupe Luz Felicita

Guadalupe Manuela Petra Pascuala de Santa Teresa

Gumersinda Clarisa Paula Fernanda

Ignacia Librada

Inés

Isabel

Joaquina Fra de Jesús

Josefa Guadalupe

Josefa Guadalupe Ana

Josefa Margarita

Josefa Margarita Ant?

Josefa Sebastiana

Juana Amada Manuela

Juana Gilberta

Juana Lucía

Juana Maria

Juana $M^{a}$. Agustina

Juana Prágiles Luz Altagracia

Juliana Crisóstoma

Justa Ant* Concepción

Lázara Dominga Liberata

Leocadia

Leocadia Restituta Ma Concepción

Leonarda de la Sma. Trinidad

Lucía Juana de Jesús

Luciana de los Reyes

Luisa Carlota Micaela

Magdalena Pascuala

Manuela Delfina Juana

Manuela de Jesús Apolonia
Manuela Joaquina Adelaida

Manuela María

Margarita Elisa

María

Ma Adelaida Hilaria

Mª Agapita Dolores Rafaela

Ma Agustina de la Sma. Trinidad*

Ma Ana

Ma Andrea Felipa de Jesús Dolores

Ma Andrea Teresa Rafaela

Ma Ángela Josefa

Ma de los Ángeles

Ma de los Ángeles Emiliana

M? de los Angeles Felicita Justina Fra

Ma de Ios Angeles Julita Elsa Marta Ignacia

Ma de los Angeles Rosa Josefa Eufemia

$M^{a}$ de Ios Ángeles Rosa Josefa Seferina

$M^{a}$ Ángeles Rosario Fra Ignacia Joaquina de la Purísima Concepción

Ma de los Angeles Valentina

$M^{a}$ de Ias Angustias Anastasia Antonia

Ma Anta Abad de Jesús

$M$ a de la Asunción*

Ma de la Asunción Ramona

Ma Asunción de la Sma. Trinidad

Ma Atilana Caritina

Ma Benigna Catarina

Ma Blasa

Ma Brigida de Jesús

Ma Bruna de Jesús Mạ José

Ma de Ia Candelaria Blasa

Ma Candelaria Severa

Ma Cándida Geralda

Ma deI Carmen Abundia

Ma del Carmen Altagracia Cruz

Ma del Carmen Cornelia

$M^{\text {a }}$ del Carmen Enriqueta Ant Fr? $^{\mathrm{a}}$ Guadalupe Juliana

Ma del Carmen Genoveva Ignacia Petra Manuela Juana Nepomucena Lucía Gonzaga

M del Carmen Hilaria Lugarda

Ma del Carmen Jacinta*

Ma del Cammen Mariana Justa Rufina

$M^{\text {a }}$ del Carmen Pía Dolores Micaela

Ma del Carmen Ramona

Ma del Carmen Rufina Gabina

Ma del Carmen Seferina Teresa Agus. tina Concepción Josefa Juana

Ma del Carmen Teofila Soledad Laura Josefa Ignacia

Ma del Carmen Teresa de Ia Luz Lázara del Corazón de Jesús

Ma Carolina Guadalupe de Jesús

Ma Cayetana Alberta

* Los nombres con asterisco atarecen dos veces (satio Concepción, qua afrarece cinco veces). 
Ma Cecilia Ignacia de los Angeles

Ma Cenobia Marciana

Ma Clara

M: Cleofas

Ma Concepción *

Ma Concepción Altagracia Teresa Ignacia de Jesús

Ma Concepción Ernestina Rosenda Fra de Paula

Ma Concepción Espiridiona

$M^{a}$ Concepción Inocencia Angela

$M^{3}$ Concepción Inocencia de Jesús

Ma de la Concepción Josefa Ignacia Federica Javiera Joaquina $\mathrm{Fr}^{\mathrm{a}}$ de Paula Ramona de la Sma. Trinidad.

Ma Concepción Leocadia

Ma Concepción Leocadia de Jesús

Ma Concepción Matiana

$M^{a}$ de la Concepción Soledad

Ma de la Consolación Frra de Paula Gertrudis Cecilia Artura

Ma Cosme Damiana

Ma de la Cruz Rómula

M* Dolores Brígida Martina

Ma Dolores Encarnación

Ma de los Dolores Fr? de Sales

M? Dolores Luz Ignacia Simona

Ma Dolores de la Luz Juana

M. Dolores Manuela Gonzaga Nicanora Fra de Paula Ignacia de Loyola Simona de Pajas Josefa de la Sma. Trinidad

Ma Dolores Maximina

Ma Dolores Natalia Inocencia

Ma Dolores Nicanor Ignacia

Ma Dolores Petronila

Ma Dolores Pomposa Agapita

Ma Dolores Pomposa Merced Guadalupe

$M^{a}$ de los Dolores Rosa

$M^{a}$ Dolores Úrsula Eulalia

Ma Dominga Fra Angeles

Ma Eduarda Calixta Fortunata

Ma Elija Bibiana de los Dolores

Ma Elija de Jesús

Ma Esteban

Ma Estefania Guadalupe

Ma Eugenia Gertrudis

Ma Eugenia Isabel

M. Evarista Simona

Ma Expectación Ausencia

Mः de la Expectación Josefa de la Sina.

Trinidad

Ma de la Expectación Timotea

M? Filogonia Juliana de la Soledad

Ma Florentina *

Ma Fra Atilana

Ma Fra Columba

Ma Fra de Paula
Ma Fra de Paula Eugenia

Ma Fra Sales Constancia

Ma Fra Severiana

M: Guadalupe*

M? Guadalupe Andrea Martina

$M^{a}$ Guadalupe de los Ángeles

Ma Guadalupe Apolonia Loreto de Jesús Ma de la Luz Telesforo Rosario Josefa Dolores

Ma Guadalupe Arnulfa

Ma Guadalupe Buenaventura Jovita

M: Guadalupe Clara

Ma Guadalupe Concepción

M: Guadalupe Dámasa

Ma Guadalupe Dolores Josefa

Ma Guadalupe Dolores Luciana

Ma Guadalupe Eulalia*

Ma Cnadalupe Enlalia Benigna

$M^{*}$ Guadalupe Felicidad

Ma Guadalupe Homobona de Jesús

$\mathrm{M}$ : Guadalupe de Jesús Dámasa Fra

M? Guadalupe de Jesús Josefa Ambrosia

M: Guadalupe de Jesús Josefa Magda. lena Isabel Matiana Apolinaria

M. Guadalupe Josefa Juana Emeteria

Eusebia del Corazón de Jesús

Ma Guadalupe Josefa Soledad

Ma Guadalupe Luisa Alfonsa Joaquina

Ma Guadalupe Melquiades

Ma Guadalupe Modesta Josefa

Ma Guadalupe Nestora

Ma Guadalupe Nicolasa de Jesús

M: Guadalupe Petra

Ma Guadalupe Refugio Feliciana Dolores

Ma Guadalupe Salomé Pascuala de Jesús

$M$ a Guadalupe Sergia

Ma Guadalupe Soledad Merced Porfiria Ant Ignacia Guadalupe de la Sma. Trinidad Josefa $F^{\mathrm{a}}$ de Paula Juana Bautista Federica Tina Ignacia Alfonsa de Liborio [= Liguori]

Ma Guadalupe Teresa Rosa

M: Guadalupe Trifonia Joaquina

M* Guadalupe Valentina Cayetana

Ma Guillerma de Jesús

Ma Herculana Severa Soledad

Ma Higinia*

M* Ildefonsa Paula

Ma Inés

Ma Isabel

Ma Isabel Felícitas

Ma Isabel Guadalupe Cecilia Soledad

Ma Jacinta

Ma Jacinta Amada

Ma de Jesús

$M^{a}$ de Jesús Amalita Soledad

Ma de Jesús Celestina Josefa 
Ma de Jesús Desideria Benigna

Ma de Jesús Dolores Fra Trinidad Concepción

$\mathrm{M}^{*}$ de Jesús Dolores Isabel Isidora Mariana Teófila Arcadia

$M^{2}$ de Jesús Dolores Viviana

$M^{a}$ a de Jesús Hipólita

Ma de Jesús Jacinta Librada

Ma de Jesús Luisa Josefa de los DoIores

Ma de Jesús Rafaela Delfina Eutimia de la Sma. Trinidad

Ma de Jesús Teodora

Ma de Jesús Trinidad Fra de Paula

Ma Josefa Camila

M Josefa Eligía Viviana Luisa Gonzaga Pascuala Juana Nepomucena

M? Josefa Gabina Fra de Paula

Ma Josefa de Jesús Luisa

Ma Josefa de Jesús Salomé

M? Josefa de Jesús Salomé Elodia Loreto Rita

M? Josefa de Jesús Saturnina

Ma Josefa Sebastiana

Ma Julia

Ma Juliana Nicanora de la Soledad

Ma Liberata Guadalupe Juliana Juana

Ma de Lorena

Ma de Lorena Tiburcia

Ma del Loreto Gorgonia

M. Loreto Guadalupe de Jesús Eduvige Rafaela

Ma deI Loreto Ignacia de la Sma. Trinidad

Ma del Loreto Josefa de Jesús Dolores Joaquina Diega

$M^{3}$ del Loreto de la Luz Adriana

Ma del Loreto Trinidad

Ma Lucía

Ma Lucía Agustina

Ma Luciana

Ma Luisa Dionisia Irene

Ma Luisa Guadalupe Erlinda Cosme Damiana

Ma de la Luz Bartola Felipa

$M^{2}$ de la Luz Celsa

$M^{a}$ de la Luz Hilaria Josefa de Jesús Paula

Ma de la Luz Isabel

$M^{2}$. de la Luz Lázara

M de la Luz Salomé Córdula

Ma Malaquías de la Soledad

M. Manuela

M? Manuela Josefa Agustina Fra Javiera Refugio

Ma Margarita

Ma Martina

Ma Martina Emilia

M: Maura Rita
Ma de la Merced Ángela Soledad Ati-

lana Aurora

Ma de la Merced Juana Ignacia

Ma de la Merced Lina de Jesús

Ma de la Merced Matiana

Ma de la Merced Soledad

M? Micaela

M. Modesta Carlota del Rosario

Ma Navarra Angelita

Ma Nestora Porfiria Fra de Paula

Ma Nicanora Anta Juana Nepomucena

Ma Nicolasa Guadalupe

$M^{a}$ de las Nieves Ángela

Ma de las Nieves Lorena

Ma de las Nieves Manuela Guadalupe

Ma Pantaleona Buenaventura Jovita

M. Pantaleona Pascuala

Ma Pantaleona Sidonia

Ma Paula

M? Paula Juventina Ant?

Ma de Paz Timotea

$M$ a de Paz Trinidad

Ma Petra Ángela Dominga

M: Petra de los Ángeles

Ma Petra Lucas

M? Petra Micaela*

M. Petronila de Ios Angeles

M? Petronila Eulalia

Ma Pilar

Ma del Pilar*

Ma del Pilar Nicasia

Ma Porfiria Josefa Rufina

Ma Prima Camila Rosalía

Ma Raquel Ignacia Trinidad

Ma Refugio

Ma Rosa Concepción

Ma del Rosario Teófila Apolinaria

$M^{3}$ de San Gonzalo Dolores Rafaela Josefa Ignacia Andrea Adelina

Ma Sebastiana Nestora

M. Severa Celestina

Ma Silvestra Martina

Ma Soledad Abundia

Ma Soledad Agustina de los Remedios

Ma Soledad Aurelia Pantaleona Nazario Celia

Ma Soledad Cleofas de Jesús

Ma Soledad Ezequía

Ma de la Soledad Felipa de Jesús

Ma Soledad $\mathrm{Fr}^{\mathrm{a}}$

Ma Soledad Fra de Borja

M: Soledad Fr? de Paula

M? Soledad Homobono Estanislao

$M$ a Soledad Ignacia Benigna

Ma Soledad Inćs

Ma Soledad Josefa Catalina

Ma Soledad Juana

Ma Soledad Leandra Agustina

Ma Soledad Lina Merced 
Ma Soledad Martina

M* Soledad Melchiora

M. Soledad Nicolasa

Ma Soledad Porfiria

M: Soledad Sabrina Mariana

Ma Soledad Santa Luz de la Sangre de Cristo

M? Soledad Telesfora

Ma Soledad Tomasa Rita

Ma Teodora Andora

Ma Teodora Andrea Avelina

Ma Teófila Guadalupe Apolonia

Ma Teresa

Ma Teresa Ángela Vicenta de Paúa

Ma Teresa de Jesús Tomasa Ant: Agustina Manuela Fra Delfina

Ma Tomasa Victoria

Ma Trinidad Catalina Paula

M. Trinidad Isabel Ponciana

M: Trinidad Rita Eduvige

Ma Úrsula Elodia

Ma Ventura Camila

Ma Vicenta Anastasia Carolina

Ma Victoria de los Ángeles

Ma Victoria Demetria Flaviana

Ma Virginia

M? Viviana de la Luz

Mariana Josefa Prisciliana Guadalupe

Matilde Martina

Máxima Maximiana Adelaida

Micaela Gudelia Emilia

Nestora Porfiria

Nicolasa Celia del Corazón de Ma Vi. centa

Nicolasa Francisca
Petra Alcántara

Petronila Desideria

Pilar Eduarda

Plácida Ma de los Dolores Encarnación del Smo. Sacramento

Rafaela Aurelia

Rafaela Evarista M: Josefa Jesús Juana

Ramona Estefanía Ma de los Remedios

Rebeca Carolina Emilia

Rómula

Rosa Eulalia Mariana Manuela Rosalía

Rosa Lamberta

Rosa Ma Bernarda

Rosa M: Concepción

Rosa M: Ramona

Rosa Ma de la Soledad

Rosa Ma Vicenta

Saturnina

Severia Antonia

Simona

Simona Maria

Simona Piedad Brígida Edelvira

Teresa Diega Aurelia Agustina

Teresa de Jesús Guadalupe Eduvige

Teresa Ventura Soledad Concepción

Tita Telesfora

Tomasa Demetria

Trinidad Agustina Guadalupe Fra Javiera

Trinidad Ma Dolores Leona Eleutina

Trinidad Ma Guadalupe Loreto Jesús Josefa Ignacia $\mathbf{F r}^{\mathrm{a}}$ de Paula Soledad Federica

Úrsula Soledad

ApÉndice IVa: 1952

Nombres masculinos

Abel Baldomero . . . I

Abraham. . . . . 1

Adalberto . . . . . 1

Adolfo. . . . . . . 1

Adrián . . . . . . 1

Adrián Ángel . . . 1

Agustín . . . . . 1

Alberto . . . . . . 3

Alberto Emilio . . . 1

Alberto Leopoldo . . 1

Alejandro . . . . . 3

Alejandro Mario . . 1

Alfonso . . . . . . 2

Alfonso Julián . . . 1

Alfredo . . . . . . 3

Álvaro. . . . . . . 1
Andrés. . . . . . . 1

Angel. . . . . . . 2

Angel Gumersindo . 1

Ángel Gonzalo . . . 1

Ángel Remigio . . . 1

Antonio . . . . . . 7

Antonio Arturo. . I

Antonio David . . . 1

Apolinar. . . . . . 1

Aquiles Félix. . . . 1

Armando. . . . . . 5

Armando Mario . . . 1

Arturo . . . . . . 10

Arturo Víctor . . . . 1

Baltasar . . . . . . 1

Benjamín. . . . . . 1
Bernardo. . . . . 2

Carlos. . . . . . . 9

Carlos Eduardo. . 1

Carlos José . . . . 2

Carlos Luis Javier. . 1

Carlos Rodolfo . . . I

Claudio Luis. . . . I

Crispin . . . . . . I

Daniel. . . . . . 1

David. . . . . . . 2

Domingo Jorge . . . I

Edmundo. . . . . . 1

Fduardo. . . . . 4

Enrique. . . . . 6

Ernesto . . . . . . 4

Esteban . . . . . 2 
Esteban Gil . . . . I Esteban Pedro. . . . 1 Ezequiel . . . . . . 1 Ezequiel Mariano . . 1 Federico. . . . . . 1 Felipe. . . . . . 2 Felipe Javier . . . . 1 Félix Emilio . . . . 1 Fernando . . . . . 10 Fernando Armando. . 1 Fernando Ignacio . . 1 Fidel . . . . . . . 1 Florentino Javier . . 1 Francisco. . . . . . 3 Francisco Javier. . . 5 Gabriel . . . . . . 1 Gaudencio . . . . . 1 Gerardo . . . . . . 1 Gerardo Antonio. : . 1 Gerardo Baltasar. . . 1 Gerardo René. . . . 1 Germán. . . . . . 1 Gonzalo . . . . . . 3 Gregorio. . . . . . 1 Guillermo . . . . 2 Guillermo Alejandro . 1 Guillermo Nicolás . . 1

Gustavo : . . . . . 1

Gustavo Adolfo . . . 1

Héctor. . . . . . . 1

Héctor Cornelio . . . 1

Héctor Guillermo. . . 1

Héctor Manuel . . . 1

Higinio . . . . . . 1

Horacio Gerardo . . 1

Hugo Alfredo. . . . 1

Humberto . . . . . 1

Ignacio . . . . . . 1

Ildefonso. . . . . . 1

Jaime. . . . . . . 4

Jaime Francisco . . . 1

Jaime Rafael . . . . 1

Javier. . . . . . 3

Javier Raúl. . . . . 1

Jesús . . . . . . . 7

Jesús Alfonso . . . . 1

Jesús Amado . . . . 1

Jesús Gerardo. . . . 1

Jesús Raúl . . . . 1

Jjoaquín . . . . . . 1

Joaquín Flaviq . . . 1

Jorge . . . . . . . 6

Jorge Alberto. . . . 1

Jorge Fernando . . . I

Jorge Óscar . . . . 1

Jorge Rafael . . . . 1

José . . . . . . . 6

José Adrián . . . . 1

José Alberto . . . . . 1
José Antonio . . . . 4

José Carlos . . . . 2

José Concepción Fidencio. . . . . . 1 José Corazón . . . . 1 José David . . . . 1 José Encarnación . . 1 José Enrique . . . . 1 José Félix . . . . . 1 José Gilberto . . . . 1 José Guadalupe . . . 1 José Gustavo . . . . 1 José Jesús . . . . . 3 José Joaquín Sebas-

tián. . . . . : . 1 José Juan . . . . 1 José Luis . . . . . 13 José Luis Antonio . . 1 José Luis Manuel . . 1 José Manuel . . . . 3 José Nicolás . . . . 1 José Pedro . . . . 1 José Primitivo. . . . 1 José Rafael. . . . . 1 José René . . . . . 1 José Trinidad . . . 1 José Ventura . . . . 1 José Walter . . . . 1 Juan . . . . . . . 7 Jaan Antonio Rubén. 1 Juan Fidel . . . . . 1 Juan Francisco . . . 1 Juan Gabriel . . . . 1 Juan Gerardo. . . . I Juan Ignacio . . . . I Juan José . . . . . 2 Juan Manuel . . . . 4 Juan Rafael . . . . 1 Juan Ramiro . . . . 1 Juan Ricardo . . . . 1 Julián. . . . . . . 1 Lázaro. . . . . . 2 Leonardo . . . . . 1 Liborio . . . . . . 1 Lucio. . . . . . . 1 Luis . . . . . . . 3

Luis Alejandro . . . I

Luis César. . . . . 1

Luis Leopoldo. . . . 1

Magdaleno . . . . 1 Manuel .. . . . . 4 Marcelo . . . . . . 1

Marco Antonio . . . 4

Marcos . . . . . . 1

Marcos Antonio . . . 1

Mario. . . . . . . 6

Mario Alberto . . . 1

Mario Francisco . . . 1

Mario Raúl . . . . 1
Martín .. . . . 1

Martín Ernesto . . . 1

Mauro Manuel . . 1

Melchor . . . . . 1

Miguel Angel. . . . 6

Miguel Héctor . . . 1

Miguel Teódulo . . . 1

Modesto. . . . . . 1

Moisés. . . . . . . 1

Néstor. . . . . . . 1

Nicolás Alfredo . . . 1

Norberto. . . . . . 2

Octavio Javier . . . 1

Odilón Alberto . . . 1

Pablo. . . . . . . 2

Pablo Ubaldo. . . . 1

Pedro. . . . . . 2

Porfirio . . . . . . 2

Próspero. . . . . . 1

Rafael. . . . . . 3

Ramón Alejandro . . 1

Raúl . . . . . . . 3

Raúl Isaías . . . . 1

Raúl Sergio . . . . 1

Raúl Trinidad . . . 1

Raymundo. . . . 3

René . . . . . . . 1

René Agustín. . . . 1

Ricardo . . . . . . 7

Ricardo Carlos . . . 1

Ricardo Enrique . . 1

Ricardo Felipe . . . 1

Ricardo Sergio . . 1

Roberto .. . . . . 2

Roberto Martiniano - 1

Roberto Pablo . . . 1

Roberto Raymundo . 1

Rodolfo . . . . . . 2

Rodolfo Ernesto . . . 1

Rodolfo Manuel . . 1

Rogelio . . . . . 2

Rubén. . . . . . 2

Sabás . . . . . . 1

Salvador, . . . . 3

Samuel . . . . . 1

Santiago Rubén . . . 1

Santiago Sergio . . . 1

Sergio. . . . . . . 3

Sergio Alfredo . . . 1

Sergio Hipólito . . . 1

Sergio Javier . . . . 1

Servando Daniel . . . 1

Silvestre . . . . . 1

Simón. . . . . . 1

Simón Bautista . . 1

Tomás. . . . . . . 1

Ubaldo . . . . . . 1

Veremundo Ricardo . 1 
Vicente Agustín . . . 1

Víctor. . . . . . . 1
Víctor Fernando . . 1

Víctor Manuel . . . 7
Wilebaldo . . . . 1

Zenás. . . . . . . 1
APÉNDICE IV $b$ : 1952

\section{Nombres femeninos}

Adela. . . . . . . 1

Adela Patricia . . . 1

Aleja . . . . . . 1

Alejandra de la Luz. 1

Alicia. . . . . . . 1

Alma Alicia Graciela. 1

Altagracia . . . . 2

Amalia . . . . . 1

Amelia . . . . . 2

Ana María . . . . . 3

Ana M: Reyna . . . 1

Ana Ma Victoria. . . 1

Angela Lourdes . . . 1

Angelina. . . . . 2

Anita . . . . . . . 1

Antonia Marcela. . . 1

Aurora . . . . . 1

Ausencia Ofelia . . . 1

Beatriz Eugenia . . . 2

Bertha María . . . 1

Bertha Ma del Refugio . . . . . 1

Blanca Andrea . . . 1

Blanca Estela . . . 5

Blanca Irene . . . . 1

Braulia . . . . . 2

Carlota . . . . . 1

Carolina . . . . . 2

Catalina. . . . . 2

Cecilia María Teresa. 1

Celia . . . . . . 1

Clara . . . . . . 1

Clara Inés . . . . . 1

Clara Lucila . . . . 1

Concepción Guillermi-

na . . . . . . 1

Cristina . . . . . 1

Cristina Antonia . . 1

Dora Patricia . . . . 1

Doria Magdalena . . 1

Elena. . . . . . . 1

Elia. . . . . . . 1

Elia Guadalupe . . . 1

Eloisa. . . . . . 1

Eloísa Genoveva. . . 1

Elsa . . . . . . . 1

Elvia . . . . . . 1

Elvia Carlota . . . . 1

Emilia . . . . . 1

Emma Olivia . . . . 1
Enriqueta . . . 1

Ernestina. . . . . 2

Esperanza . . . . 4

Estefania. . . . . 1

Estela. . . . . . 3

Esther. . . . . . . 2

Eulalia Angelina . . 1

Eulalia Yolanda. . . 1

Eva. . . . . . . . 1

Eva Eugenia. . . . . 1

Evangelina Elvia . . 1

Evelia Enriqueta . . 1

Felicitas Patricia . . 1

Florencia. . . . . . 1

Francisca. . . . . 1

Georgina. . . . . 1

Gloria. . . . . 2

Gloria Elena . . . . 1

Gloria Modesta . . . 1

Graciela . . . . . 5

Guillermina. . . . 3

Hermelinda. . . . . 1

Hilda . . . . . . 1

Imelda Reyna. . . . 1

Irma . . . . . . . 4

Irma Bertha . . . . 1

Irma Estela Guadalu-

pe . . . . . . 1

Irma Gloria . . . . 1

Irma Silvia Marina. . 1

Irma Socorro . . . . 1

Josefa. . . . . . . 1

Josefina . . . . . 2

Jovita. . . . . . . 1

Juana. . . . . . . 2

Juana María . . . . 1

Juana Noemí . . . . 1

Judith . . . . . . 1

Judith Leticia Eliza-

beth . . . . . 1

Julia . . . . . . . 1

Juliana . . . . . . 1

Laura. . . . . . . 1

Laura Araceli. . . . 1

Laura Elena . . . . 1

Laura Rafaela . . . 1

Laura Romina . . . 1

Laureanabea . . . . 1

Leticia . . . . . . 2

Lidia Hermelinda . . 1
Lilia . . . . . 3

Lilia del Carmen. . . 1

Lilia Griselda. . . . 1

Lilia Guadalupe . . . I

Lorenza Trinidad . . 1

Lourdes Josefina. . . 1

Lucina Irma . . . . 1

Lucrecia . . . . . 1

Luisa María Ángeles. 1

Luz Angelina . . . . 1

Magdalena . . . . 2

Marcela . . . . . 3

Margarita . . . . 4

María. . . . . 1

Ma Alma Patricia . . 1

Ma Alva Adela . . . 1

Ma Amalia . . . . 1

Ma Anastasia Francis-

ca . . . . . . . 1

Ma (de los) Ángeles. 4

Ma Antonia . . . . 1

Ma Antonieta. . . . 2

Ma Ardelia. . . . . 1

Ma Asunción . . . . 1

Ma Aurora. . . . . 1

Ma Beatriz Yolanda. 1

Ma Blanca . . . . . 1

Ma Blanca Agapita . 1

M. Candelaria . . . 1

Ma del Carmelo . . . 1

Ma del Carmen . . . 9

Ma Carmen Liboria

Inés. . . . . . 1

Ma Catalina . . . . 1

Ma Clara . . . . . 1

Ma Concepción . . . 4

Ma Constancia . . . 1

Ma Consuelo . . . . 1

Ma Cristina . . . . 1

M* Delia Isabel . . 1

Ma Elena . . . . . 4

Ma Elena Catalina . . 1

M. Elizabeth . . . . 1

M? Elodia . . . . . 1

Ma Encarnación . . . 1

Ma Enriqueta . . . . 1

Ma Ermelinda . . . 1

Ma Esperanza . . . . 1

Ma Estela . . . . . 1

Ma Esther . . . . 5 
Ma Eugenia . . . . 2

M: Eugenia Ofelia . . I

Ma Eugenia de la Virgen del Sagrado Corazón . . . . . . 1 Ma Eva . . . . . . 1

M? Graciela . . . . 1

M Graciela Paula . 1

M. Guadalupe . ., 17

Ma Guadalupe Raquel.

Ma Hortensia . . . . I

Ma Imilde . . . . . 1

Ma Inés . . . . . . 1

Ma Inocencia . . . . 1

Ma Irene . . . . . 1

Ma Irma. . . . . . 1

Ma Irma Marina . . l 1

Ma Isabel . . . . . 7

Ma de Jesús . . . . 3

Ma de Jesús Edit . . 1

M? Jesús Patricia. . 1

Ma Laura . . . . . I

Ma de Lourdes . . , 5

Ma de Lourdes Gracie-

la. . . . . . . . 1

M Lucila . . . . . 1

Ma Luisa . . . . . 2

Ma de la Luz . . . 6

Ma Magdalena . . . 1

Ma Martha Yolanda . I

Ma Minerva . . . . 1

Ma Miriam . . . . 1

M? Natividad . . . . I

Ma Ofelia . . . . . 1

Ma Pamela . . . . I

Ma Patricia . . . . 1

Mะ Paula . . . . . I

Ma Paula Yadira . . l
M del Perpetuo Socorro . . . . . . 1 Ma Pilar . . . . . I

Ma Remedios . . . . 2

Ma Reyna . . . . . 1

Ma Rosa Lourdes. . . I

Ma (del) Rosario . . 3

Ma Rosela . . . . . 1

Ma Ruth. . . . . . 1

Ma Santa . . . . . 1

Ma del Socorro . . . 2

Ma Soledad . . . . I

Ma Teresa . . . . . 7

Ma Teresita de Jesús. i

Ma Trinidad Ofelia, I

Ma Victoria . . . . 2

Ma Yolanda . . . . 1

Mariana Guadalupe . I

Maricela. . . . . . 4

Marina. . . . . . 1

Marina del Carmen . I

Marina Inés . . . . 1

Marta Elsa . . . . . I

Marta Susana . . . . 1

Martha . . . . . . 3

Martha Rosa . . . . I

Martha Elva . . . 1

Martina . . . . . . 1

Matilde Leticia . . . 1

Mercedes Amalia. . . 1

Mercedes Genoveva . 1

Micaela . . . . . 2

Miriam Socorro . . . 1

Modesta . . . . . .

Natalia María Guada-

lupe. . . . . . . 1

Noemí. . . . . . . 1

Nora Yolanda . . . 1
Ofelia. . . . . . 1

Patricia . . . . . 1

Paula Lourdes . . . 1

Prudencia Teresa . . I

Ramona . . . . . . 1

Rebeca . . . . . 2

Rita Eugenia . . . . I

Rosa . . . . . . . 1

Rosa Fátima Lucía. . 1

Rosa Lilia . . . . . 1

Rosa María . . . . 7

Rosa Ma Martha. . . 1

Rosa Martha lima . I

Rosa Natalia . . . . 1

Rosa Vicenta . . . I

Rosalía . . . . . . 1

Rosalinda . . . . . I

Sara . . . . . . 4

Sara Clotilde . . . . 1

Sara Matilde . . . 1

Sara Mercedes. . . . 1

Silveria Guadalupe. . 1

Silvia. . . . . . 8

Socorro . . . . . . 1

Sofía . . . . . . . 2

Sofía Francisca . . . I

Soledad . . . . . . 1

Susana . . . . . 2

Susana Lilia . . . . 1

Teresa . . . . . 2

Teresita del Niño Je-

sús . . . . . . 1

Victoria Eugenia. . . 1

Victorina . . . . . I

Violeta Eloísa . . . 1

Virginia . . . . . 3

Yolanda . . . . . . 5

Yolanda Irma . . . 1

Yolanda Margarita . I 